\title{
THE FRINGE REGION TECHNIQUE AND THE FOURIER METHOD USED IN THE DIRECT NUMERICAL SIMULATION OF SPATIALLY EVOLVING VISCOUS FLOWS*
}

\author{
JAN NORDSTRÖM ${ }^{\dagger}$, NIKLAS NORDIN ${ }^{\ddagger}$, AND DAN HENNINGSON ${ }^{\S}$
}

\begin{abstract}
To eliminate the problem with artificial boundary conditions and facilitate the use of Fourier methods, the fringe region (or filter, damping layer, absorbing layer, sponge layer) technique has been used in direct simulations of transitional and turbulent boundary layers. Despite the fact that good computational results have been obtained with this technique, it is not fully understood. The analysis in this paper indicates that the primary importance of the fringe region technique is to damp out the deviation associated with large scales in the direction normal to the wall. The lack of boundary conditions is compensated by the knowledge of an exact solution in the fringe region of the computational domain. The upstream influence from the fringe region is small. Numerical experiments verifying the theoretical predictions are presented.
\end{abstract}

Key words. initial boundary value problem, Navier-Stokes equations, Fourier methods

AMS subject classifications. 35B10, 35B27, 35B30, 76D05

PII. S1064827596310251

1. Introduction. In many computational problems one is faced with infinite or semi-infinite domains, which for computational reasons must be made finite. The computational domain is normally reduced to a finite domain by an artificial boundary. Information from the solution in the exterior domain to the solution in the computational domain is transferred via the artificial boundary conditions. The type of knowledge about the exterior solution and how to transfer this knowledge to the artificial boundary conditions is, roughly speaking, what separates the different types of artificial boundary conditions in the literature. For a discussion on these matters see Gustafsson and Kreiss [1]; a comprehensive review on artificial boundary conditions is given by Givoli [2].

The artificial boundary conditions must lead to a well-posed continuous problem and augmented with numerical boundary conditions, a stable discrete problem. For a discussion on well-posedness and stability, see Kreiss [3] and Gustafsson, Kreiss, and Sundström [4]. To eliminate the difficulties with boundary conditions and facilitate the use of Fourier methods, the fringe region technique, originally introduced by Spalart [5], has been used in direct simulations of transitional and turbulent boundary layers; see Bertolotti, Herbert, and Spalart [6], Spalart and Watmuff [7], Lundbladh et al. [8], and Berlin, Lundbladh, and Henningson [9]. The computational domain is divided into one useful region and one fringe region. An extra forcing function is added to the momentum equations in the fringe region to compensate for the periodicity of

* Received by the editors October 2, 1996; accepted for publication (in revised form) December 14, 1997; published electronically March 22, 1999. This work was sponsored by the NUTEK Fluid Dynamics Program.

http://www.siam.org/journals/sisc/20-4/31025.html

${ }^{\dagger}$ FFA, Aeronautical Research Institute of Sweden, Box 110 21, SE-161 11 Bromma, Sweden, and Department of Scientific Computing, Uppsala University, SE-751 04 Uppsala, Sweden (nmj@ffa.se).

$\ddagger$ FFA, Aeronautical Research Institute of Sweden, Box 110 21, SE-161 11 Bromma, Sweden. Present address: Department of Thermo and Fluid Dynamics, Chalmers University of Technology, SE-412 96 Gothenburg, Sweden (nordin@tfd.chalmers.se).

§FFA, Aeronautical Research Institute of Sweden, Box 110 21, SE-161 11 Bromma, Sweden, and Department of Mechanics, Royal Institute of Technology, SE-100 44 Stockholm, Sweden (hnd@ffa.se). 
the problem. It is assumed that the nonphysical phenomena occurring in the fringe region do not invalidate the solution in the remaining part of the computational domain. Information about the exterior solution is transferred to the computational domain via the forcing function.

The Fourier-fringe region method for direct simulations of transitional and turbulent boundary layers is efficient and very accurate. In Bertolotti, Herbert, and Spalart [6] and Högberg and Henningson [10], for example, it is demonstrated that growth rates of sensitive instability modes are predicted very accurately when evaluated from direct numerical simulations utilizing the fringe region technique. Despite the fact that good computational results have been obtained using this technique, it is not fully understood. The purpose of this paper is to analyze why an already existing method works well; it is not our ambition to propose a new computational method.

Techniques similar to the Fourier-fringe region technique have also been used in conjunction with other types of discretization methods and in other fields of physics. Kloker, Konzelmann, and Fasel [11] suppressed the vorticity disturbances close to the outflow boundary in a transitional boundary layer by means of a weighting function. Colonious, Lele, and Moin [12] combined a stretched grid with a filter to avoid reflections from the outflow boundary. Karni [13] modified the governing equations in the fringe region to accelerate convergence to steady state. For examples of other fields of computational physics where the fringe region technique has been used, see Berenger [14] and Davies [15].

The remainder of this paper will proceed as follows. An exact linear initial boundary value problem for the deviation between the approximate solution computed using the fringe region technique and an exact solution is derived in section 2 and analyzed in section 3. Two constant coefficient problems related to the linear problem are derived in section 4 . The constant coefficient problems are analyzed in section 5 . Numerical experiments related to the theoretical results in section 5 are presented in section 6 . In section 7 , we sum up and draw conclusions.

2. The linear problem. The direct numerical simulation of a boundary layer will be considered. The solution computed by the fringe region technique will be compared with an exact solution $\mathbf{u}(x, y, t)$ to the Navier-Stokes equations. The solution is given by

$$
\begin{aligned}
\mathbf{u}_{t}^{1} & =P\left(\mathbf{u}^{1}\right) \mathbf{u}, & & 0 \leq y, \quad t \geq 0, \\
\nabla \cdot \mathbf{u}^{1} & =0, & & 0 \leq y, \quad t \geq 0,
\end{aligned}
$$

where $\mathbf{u}=\left(\mathbf{u}^{1}, u_{3}\right), \mathbf{u}^{1}=\left(u_{1}, u_{2}\right)$, and

$$
\begin{gathered}
P\left(\mathbf{u}^{1}\right)=-\left(A\left(\mathbf{u}^{1}\right) \frac{\partial}{\partial x}+B\left(\mathbf{u}^{1}\right) \frac{\partial}{\partial y}\right)+\epsilon\left(C \frac{\partial^{2}}{\partial x^{2}}+D \frac{\partial^{2}}{\partial y^{2}}\right), \quad \nabla \cdot \mathbf{u}^{1}=\frac{\partial u_{1}}{\partial x}+\frac{\partial u_{2}}{\partial y} \\
A\left(\mathbf{u}^{1}\right)=\left(\begin{array}{ccc}
u_{1} & 0 & 1 \\
0 & u_{1} & 0
\end{array}\right), \quad B\left(\mathbf{u}^{1}\right)=\left(\begin{array}{ccc}
u_{2} & 0 & 0 \\
0 & u_{2} & 1
\end{array}\right), \\
C=\left(\begin{array}{ccc}
1 & 0 & 0 \\
0 & 1 & 0
\end{array}\right), \quad D=\left(\begin{array}{ccc}
1 & 0 & 0 \\
0 & 1 & 0
\end{array}\right) .
\end{gathered}
$$

The dependent variables and parameter $u_{1}, u_{2}, u_{3}$, and $\epsilon$ are, respectively, the $x$ and $y$ components of the velocity, the pressure, and the inverse of the Reynolds number. The problem (2.1) has to be augmented with boundary conditions and an 

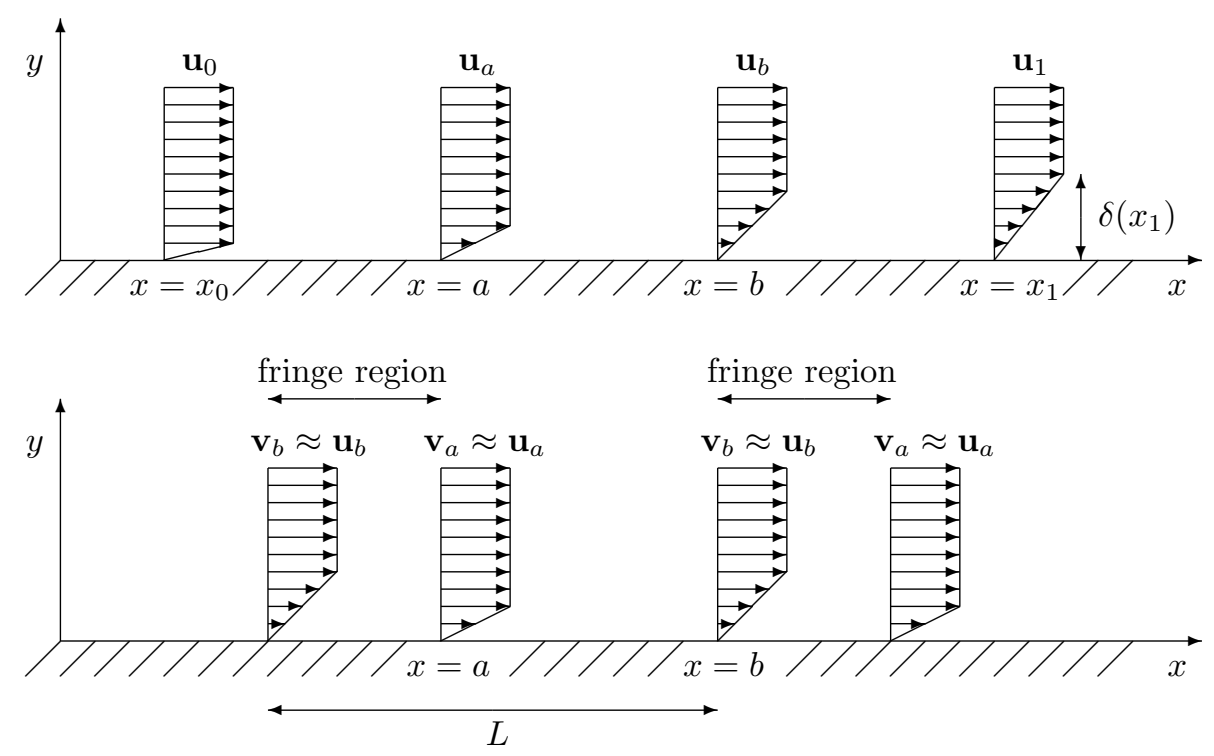

FIG. 2.1. A schematic view of the exact solution $\mathbf{u}$ and the periodic solution $\mathbf{v}$.

initial condition. At this point it suffices to mention that the solution approaches a constant value for large $y$ and that we have a no-slip condition at the wall $y=0$, i.e.,

$$
\mathbf{u}^{1}(x, 0, t)=0, \quad \lim _{y \rightarrow \infty} \mathbf{u}^{1}(x, y, t)=\mathbf{u}_{\infty}^{1} .
$$

In the fringe region technique one approximates $\mathbf{u}$ in the domain $[x, y] \in \Omega_{0}$, $\Omega_{0}=[a, b] \times[0, \infty[$ by the periodic solution $\mathbf{v}$ to

$$
\begin{aligned}
\mathbf{v}_{t}^{1} & =P\left(\mathbf{v}^{1}\right) \mathbf{v}-\lambda(x)\left(\mathbf{v}^{1}-\dot{\mathbf{u}}^{1}\right), & & 0 \leq y, \quad t \geq 0, \\
\nabla \cdot \mathbf{v}^{1} & =0, & & 0 \leq y, \quad t \geq 0,
\end{aligned}
$$

where $\mathbf{v}=\left(\mathbf{v}^{1}, v_{3}\right), \mathbf{v}^{1}=\left(v_{1}, v_{2}\right)$ satisfies condition (2.2). The forcing function $-\lambda(x)\left(\mathbf{v}^{1}-\dot{\mathbf{u}}^{1}\right)$ is introduced in order to compensate for the periodicity of $\mathbf{v}(x, y, t)=$ $\mathbf{v}(x+L, y, t), L>b-a . \mathbf{u}^{1}$ is an approximation of the exact solution $\mathbf{u}^{1}$ close to $x=a$. The approximation of $\mathbf{u}$ by $\mathbf{v}$ is schematically depicted in Figure 2.1 and the fringe function $\lambda(x)$ used in the numerical experiments presented below is shown in Figure 2.2. $\lambda(x)$ has a flat maximum and is nonzero in a portion of the computational domain with length $\alpha$ called the fringe region.

Let $\mathbf{w}=\mathbf{u}-\mathbf{v}$. By subtracting (2.3) from (2.1) with artificial boundaries introduced at $x=x_{0}, x_{1}$ we obtain

$$
\begin{aligned}
& \mathbf{w}_{t}^{1}=P\left(\mathbf{v}^{1}\right) \mathbf{w}-E\left(\mathbf{u}_{x}^{1}, \mathbf{u}_{y}^{1}\right) \mathbf{w}^{1}+F(x, y, t), \quad[x, y] \in \Omega_{+}, \quad t \geq 0, \\
& \nabla \cdot \mathbf{w}^{1}=0, \quad[x, y] \in \Omega_{+}, \quad t \geq 0, \\
& \mathbf{w}=f(x, y), \quad[x, y] \in \Omega_{+}, \quad t=0, \\
& L_{0} \mathbf{w}=g_{0}(y, t), \quad x=x_{0}, 0 \leq y, \quad t \geq 0, \\
& L_{1} \mathbf{w}=g_{1}(y, t), \quad x=x_{1}, 0 \leq y, \quad t \geq 0, \\
& \mathbf{w}^{1}=0, \quad x \in\left[x_{0}, x_{1}\right], \quad y=0, \quad t \geq 0, \\
& \mathbf{w}^{1} \rightarrow 0, \quad x \in\left[x_{0}, x_{1}\right], \quad y \rightarrow \infty, \quad t \geq 0,
\end{aligned}
$$




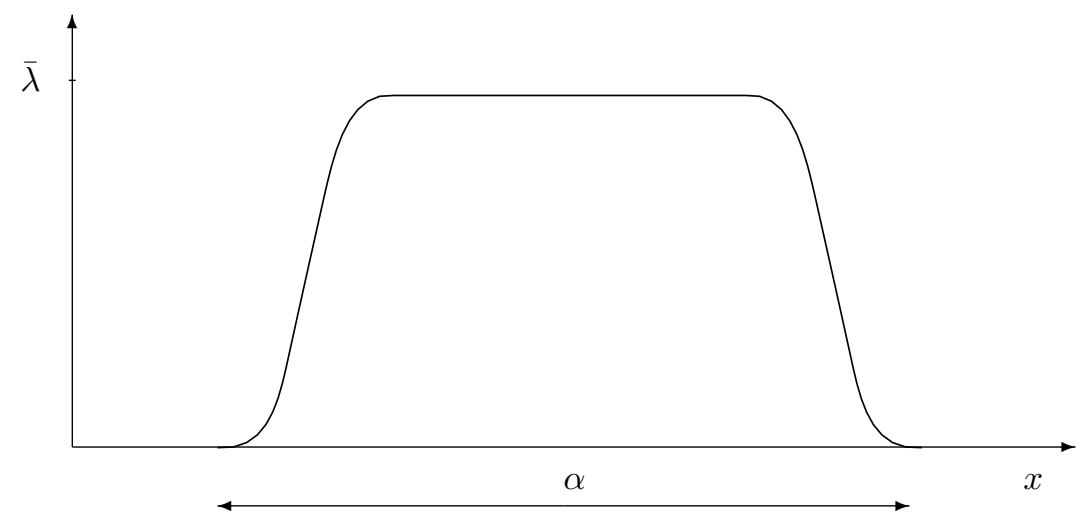

FIG. 2.2. An example of the fringe function.

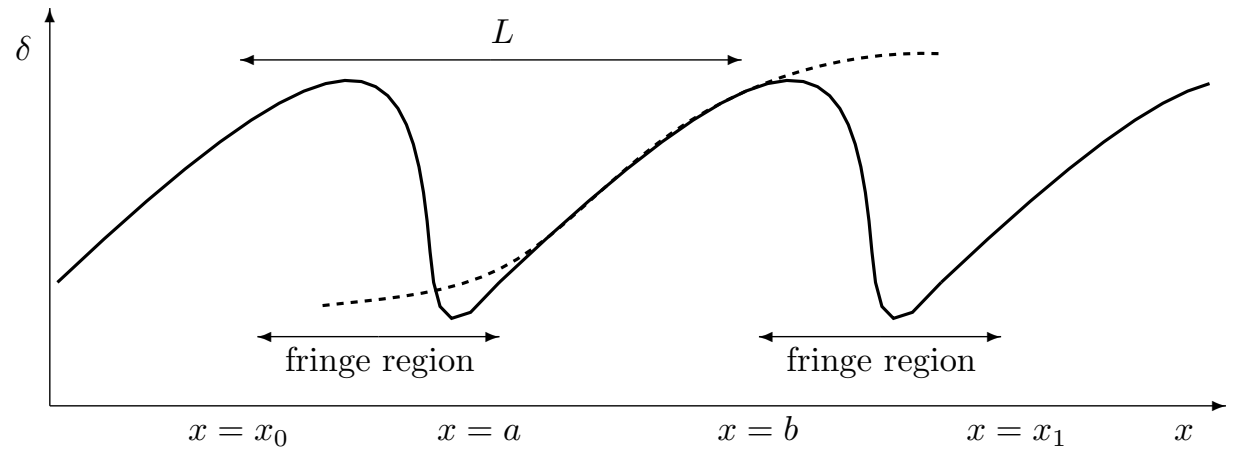

FIG. 2.3. Boundary layer thickness as a function of $\mathbf{u}$ (dotted line) and $\mathbf{v}$ (solid line).

where $\mathbf{w}=\left(\mathbf{w}^{1}, p\right), \mathbf{w}^{1}=(u, v)$, and $\Omega_{+}=\left[x_{0}, x_{1}\right] \times\left[0, \infty\left[. L_{0}, L_{1}\right.\right.$ are the boundary operators at $x_{0}, x_{1}$, respectively, and

$$
\begin{gathered}
F=\lambda(x)\left(\mathbf{v}^{1}-\dot{\mathbf{u}}^{1}\right)=-\lambda(x) \mathbf{w}^{1}+\lambda(x) \Delta \mathbf{u}^{1}, \quad \Delta \mathbf{u}^{1}=\left(\mathbf{u}^{1}-\dot{\mathbf{u}}^{1}\right), \\
E\left(\mathbf{u}_{x}^{1}, \mathbf{u}_{y}^{1}\right)=\left(\begin{array}{cc}
\left(u_{1}\right)_{x} & \left(u_{1}\right)_{y} \\
\left(u_{2}\right)_{x} & \left(u_{2}\right)_{y}
\end{array}\right) .
\end{gathered}
$$

For $v_{1}>0, x=x_{0}$ is an artificial inflow boundary and $x=x_{1}$ is an artificial outflow boundary.

Remark. The nonlinearity in (2.4) has been avoided by using $\mathbf{u}^{1}-\mathbf{w}^{1}=\mathbf{v}^{1}$ in the operator $P$. Both $\mathbf{u}$ and $\mathbf{v}$ are assumed to be bounded known functions in this paper.

3. Analysis of the linear problem. The problem (2.4) is an initial boundary value problem with variable coefficients and a full collection of data $\left(F, f, g_{0}, g_{1}\right)$. The data cannot be considered small; hence energy estimates will not imply that $\mathbf{v}$ is an accurate approximation of $\mathbf{u}$. However, estimates of the solution in the region $\Omega_{2}=[a, b] \times\left[0, \infty\left[\right.\right.$ in terms of the solution in the fringe regions $\Omega_{1}=\left[x_{0}, a\right] \times[0, \infty[$ and $\Omega_{3}=\left[b, x_{1}\right] \times[0, \infty[$ and the data are of interest; see Figures 2.1 and 2.3.

The proof of the following theorem is given in Appendix C. 
THEOREM 3.1. The solution to the problem (2.4) satisfies

$$
\begin{aligned}
\left\|\mathbf{w}^{1}\right\|_{2}^{2} & +2 \epsilon \int_{0}^{T}\left(\left\|\mathbf{w}_{x}^{1}\right\|_{2}^{2}+\left\|\mathbf{w}_{y}^{1}\right\|_{2}^{2}\right) e^{\tilde{\lambda}_{2}(T-t)} d t \\
\leq & \|f\|^{2} e^{\tilde{\lambda}_{2} T}+\int_{0}^{T}\left(\|g\|_{\Gamma}^{2}+\tilde{\lambda}_{3}\left\|\Delta \mathbf{u}^{1}\right\|_{3}^{2}\right) e^{\tilde{\lambda}_{2}(T-t)} d t \\
& -\left(\left\|\mathbf{w}^{1}\right\|_{1}^{2}+2 \epsilon \int_{0}^{T}\left(\left\|\mathbf{w}_{x}^{1}\right\|_{1}^{2}+\left\|\mathbf{w}_{y}^{1}\right\|_{1}^{2}\right) e^{\tilde{\lambda}_{2}(T-t)} d t\right) \\
& -\left(\left\|\mathbf{w}^{1}\right\|_{3}^{2}+2 \epsilon \int_{0}^{T}\left(\left\|\mathbf{w}_{x}^{1}\right\|_{3}^{2}+\left\|\mathbf{w}_{y}^{1}\right\|_{3}^{2}\right) e^{\tilde{\lambda}_{2}(T-t)} d t\right) \\
& -\int_{0}^{T}\left(\left(\tilde{\lambda}_{1}+\tilde{\lambda}_{2}\right)\left\|\mathbf{w}^{1}\right\|_{1}^{2}+\tilde{\lambda}_{2}\left\|\mathbf{w}^{1}\right\|_{3}^{2}\right) e^{\tilde{\lambda}_{2}(T-t)} d t
\end{aligned}
$$

where

$$
\begin{gathered}
\|f\|^{2}=\int_{\Omega_{+}}|f|^{2} d x d y, \quad\|g\|_{\Gamma}^{2}=\int_{0}^{\infty}\left(\left|v_{1}\right|\left|g_{0}\right|^{2}\right)_{x=x_{0}}+\left(\left|v_{1}\right|\left|g_{1}\right|^{2}\right)_{x=x_{1}} d y, \\
\left\|\Delta \mathbf{u}^{1}\right\|_{3}^{2}=\int_{\Omega_{3}}\left|\Delta \mathbf{u}^{1}\right|^{2} d x d y, \quad\left\|\mathbf{w}^{1}\right\|_{i}^{2}=\int_{\Omega_{i}}\left|\mathbf{w}^{1}\right|^{2} d x d y, \\
\lambda_{i}(t)=\max _{\Omega_{i}} \kappa, \quad \kappa=\sqrt{\left(\left(u_{1}\right)_{y}+\left(u_{2}\right)_{x}\right)^{2}+4\left(\left(u_{1}\right)_{x}\right)^{2}}, \quad i=1,2,3, \\
\tilde{\lambda}_{1}=\min _{0 \leq t \leq T} \frac{\left\|\sqrt{\lambda_{1}} \mathbf{w}^{1}\right\|_{1}^{2}}{\left\|\mathbf{w}^{1}\right\|_{1}^{2}}, \quad \tilde{\lambda}_{2}=\max _{0 \leq t \leq T} \frac{\left\|\sqrt{\lambda_{2}} \mathbf{w}^{1}\right\|_{2}^{2}}{\left\|\mathbf{w}^{1}\right\|_{2}^{2}}, \quad \tilde{\lambda}_{3}=\max _{0 \leq t \leq T} \frac{\left\|\sqrt{\lambda_{3}} \Delta \mathbf{u}^{1}\right\|_{3}^{2}}{\left\|\Delta \mathbf{u}^{1}\right\|_{3}^{2}} .
\end{gathered}
$$

The additional terms on the right-hand side of (3.1) due to the forcing function in the fringe region technique are

$$
-\int_{0}^{T}(\underbrace{\left(\tilde{\lambda}_{1}+\tilde{\lambda}_{2}\right)\left\|\mathbf{w}^{1}\right\|_{1}^{2}}_{\text {from } \Omega_{1}}+\underbrace{\left(\tilde{\lambda}_{2}\left\|\mathbf{w}^{1}\right\|_{3}^{2}-\tilde{\lambda}_{3}\left\|\Delta \mathbf{u}^{1}\right\|_{3}^{2}\right)}_{\text {from } \Omega_{3}}) e^{\tilde{\lambda}_{2}(T-t)} d t .
$$

Roughly speaking, (3.3) indicates that $\left|\mathbf{w}^{1}\right|$ in $\Omega_{2}$ is reduced by the events in the fringe region $\Omega_{1}$. The effect by the fringe region $\Omega_{3}$ is unclear and depends on the sign of $\tilde{\lambda}_{2}|| \mathbf{w}^{1}\left\|_{3}^{2}-\tilde{\lambda}_{3}|| \Delta \mathbf{u}^{1}\right\|_{3}^{2}$. More information on the spatial distribution of $\left|\mathbf{w}^{1}\right|$ cannot be obtained from (3.1). A more detailed analysis is necessary in order to determine that distribution and how much of the error $\mathbf{w}^{1}$ that "leaks out" of the fringe regions $\Omega_{1}$ and $\Omega_{3}$ into the supposedly useful region $\Omega_{2}$. This analysis will be the topic in the rest of this paper.

4. The constant coefficient problems. A number of approximations of (2.4) are necessary to facilitate a more detailed analysis. These approximations must be carefully chosen such that (i) an analysis of the simplified problem is possible and (ii) the conclusions drawn from the analysis of the simplified problem are relevant for the full problem (2.4). Two half-plane problems describing the possibility to force $\mathbf{w}$ toward zero inside the fringe region (the inflow problem) and the upstream influence of the fringe region (the outflow problem) will be constructed.

First of all, the less complicated boundary operators, $L_{0} \mathbf{w}=\mathbf{w}^{1}$ and $L_{1} \mathbf{w}=\mathbf{w}^{1}$, will be considered. These boundary operators also lead to well-posedness of (2.4). 
The boundary data $g_{0}=\mathbf{u}^{1}\left(x_{0}, y, t\right)-\mathbf{v}^{1}\left(x_{0}, y, t\right), g_{1}=\mathbf{u}^{1}\left(x_{1}, y, t\right)-\mathbf{v}^{1}\left(x_{1}, y, t\right)$, and the forcing function $F$ (see (2.5)) are zero at the wall $y=0$. Let $\delta(x)$ denote the boundary layer thickness; see Figures 2.1 and 2.3. It is reasonable to assume that $F, g_{0}, g_{1}$ also vanish for $y>\delta\left(x_{1}\right)=\delta_{1}$, i.e., we have

$$
F(x, y, t)=0, \quad g_{0}(y, t)=0, \quad g_{1}(y, t)=0, \quad y=0, \quad \text { and } \quad y>\delta_{1}
$$

for the problem (2.4).

The domain $\Omega_{+}=\left[x_{0}, x_{1}\right] \times\left[0, \infty\left[\right.\right.$ in (2.4) is expanded to $\left.\Omega=\left[x_{0}, x_{1}\right] \times\right]-\infty$, $+\infty$ [ in order to enable the use of Fourier transforms in the $y$ direction. The relation (4.1) now corresponds to

$$
F(x, y, t)=0, \quad g_{0}(y, t)=0, \quad g_{1}(y, t)=0, \quad|y| \geq \delta, \quad 0<\delta<\infty .
$$

Furthermore, identical initial data in (2.1) and (2.3) are assumed, i.e., $f=0$. An additional simplification is obtained by freezing the variable coefficients at constant states $\overline{\mathbf{u}}=(\bar{u}, \bar{v})$ and $\bar{\lambda}$. This yields $P\left(\mathbf{v}^{1}\right)=\bar{P}, E\left(\mathbf{u}_{x}^{1}, \mathbf{u}_{y}^{1}\right)=0$, and $F=\bar{\lambda}\left(\mathbf{v}^{1}-\dot{\mathbf{u}}^{1}\right)$. Note that inside the fringe region $\lambda(x)=\bar{\lambda}$; see Figure 2.2.

Remark. Investigating a linear problem by analyzing the corresponding constant coefficient problem and neglecting the terms $E\left(\overline{\mathbf{u}}_{x}^{1}, \overline{\mathbf{u}}_{y}^{1}\right) \mathbf{w}$ is a common and normally fruitful procedure; see Kreiss and Lorenz [16] and Johansson [17]. However, in special cases these terms can be important; see Nordström [18] for an example.

Let us first consider the inflow problem. Let $\Omega$ be such that the fringe region is located to the utmost left in the computational domain and let $x_{1}=a$ be located at the very end of the fringe region; see Figures 2.3 and 4.1. At the inflow boundary $g_{0}=\mathbf{u}^{1}\left(x_{0}, y, t\right)-\mathbf{v}^{1}\left(x_{0}, y, t\right)=g$. With the exact solution $\mathbf{u}^{1}$ known in the region close to the inflow boundary, we can put $\dot{\mathbf{u}}^{1}=\mathbf{u}^{1}$, which yields $F=-\bar{\lambda} \mathbf{w}^{1}$. Now let $x_{0}=0$ and $x_{1} \rightarrow \infty$; the Fourier transformed inflow problem becomes

$$
\begin{aligned}
& \hat{\mathbf{w}}_{t}^{1}=\hat{\bar{P}} \hat{\mathbf{w}}-\bar{\lambda} \hat{\mathbf{w}}^{1}, \quad x \geq 0, \quad t \geq 0, \\
& \hat{\nabla} \cdot \hat{\mathbf{w}}^{1}=0, \quad x \geq 0, \quad t \geq 0, \\
& \hat{\mathbf{w}}=0, \quad x \geq 0, \quad t=0, \\
& \hat{\mathbf{w}}^{1}=\hat{g}, \quad x=0, \quad t \geq 0, \\
& \left|\hat{\mathbf{w}}^{1}\right|<\infty, \quad x \rightarrow+\infty, \quad t \geq 0 .
\end{aligned}
$$

The analysis of (4.3) will yield an estimate of $|\hat{\mathbf{w}}|$ for $x>0$. Note that a necessary (but not sufficient) condition to obtain a small $|\hat{\mathbf{w}}|$ for $x>0$ is that the exact solution $\mathbf{u}^{1}$ is known in a region close to the inflow boundary. It is not sufficient to know $\mathbf{u}^{1}$ at one specific $x$ location. Unless one can put $\mathbf{u}^{1}=\mathbf{u}^{1}$, a forcing term will remain in (4.3). That term will lead to solutions $|\hat{\mathbf{w}}|=\mathcal{O}\left(\bar{\lambda}\left|\mathbf{u}^{1}-\dot{\mathbf{u}}^{1}\right|\right)$ for $x>0$.

Next we consider the outflow problem. Let $\Omega$ be such that the fringe region is located to the utmost right in the computational domain and let $x_{0}<b$ be located upstream of the fringe region; see Figures 2.3 and 4.2. At the outflow boundary $g_{1}=\mathbf{u}^{1}\left(x_{1}, y, t\right)-\mathbf{v}^{1}\left(x_{1}, y, t\right)=g$. The forcing function cannot be made zero in this case; we have $F=\bar{\lambda}\left(\mathbf{v}^{1}(x, y, t)-\mathbf{u}^{1}(x, y, t)\right)=\bar{\lambda}\left(\mathbf{v}^{1}(x, y, t)-\mathbf{u}^{1}(x-L, y, t)\right)=\bar{\lambda} \Delta \mathbf{u}^{1}$, $\Delta \mathbf{u}^{1}=(\Delta u, \Delta v)$. Now let $x_{0} \rightarrow-\infty$ and $x_{1}=0$; the Fourier transformed outflow problem becomes

$$
\begin{aligned}
& \hat{\mathbf{w}}_{t}^{1}=\hat{\bar{P}} \hat{\mathbf{w}}+\hat{F}, \quad x \leq 0, \quad t \geq 0, \\
& \hat{\nabla} \cdot \hat{\mathbf{w}}^{1}=0, \quad x \leq 0, \quad t \geq 0, \\
& \hat{\mathbf{w}}=0, \quad x \leq 0, \quad t=0, \\
& \hat{\mathbf{w}}^{1}=\hat{g}, \quad x=0, \quad t \geq 0, \\
& \left|\hat{\mathbf{w}}^{1}\right|<\infty, \quad x \rightarrow-\infty, \quad t \geq 0 .
\end{aligned}
$$




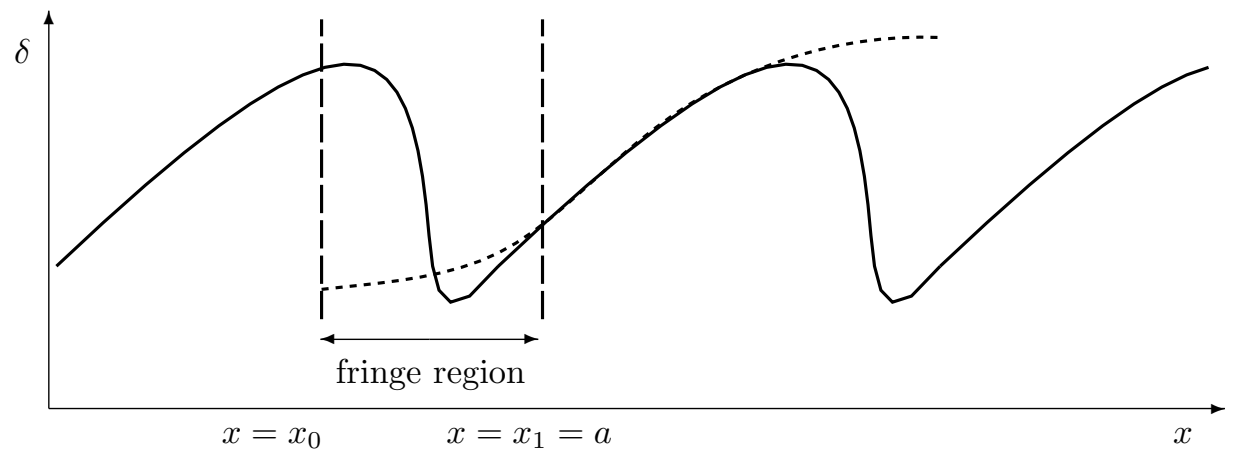

FIG. 4.1. The inflow problem with artificial boundaries.

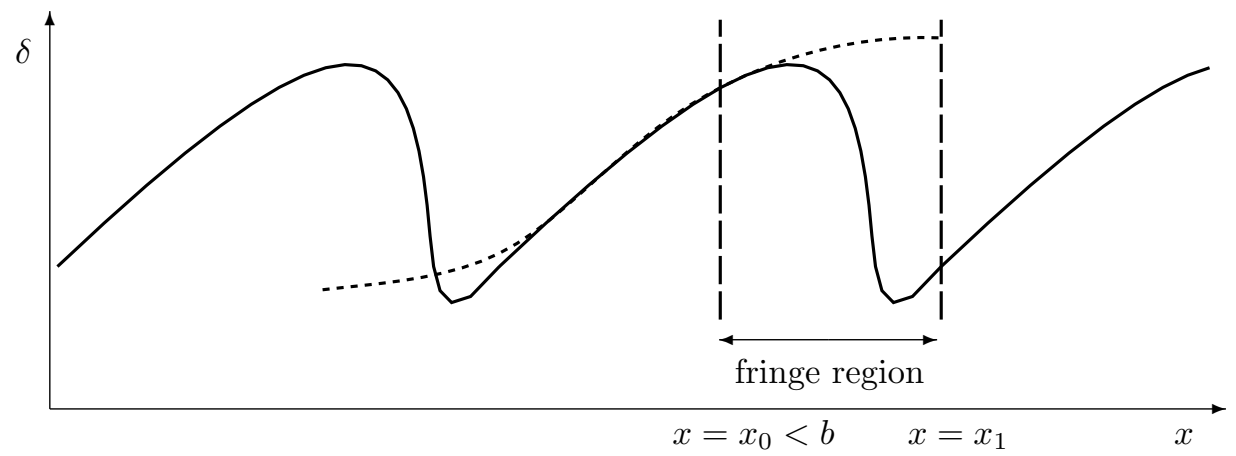

FIG. 4.2. The outflow problem with artificial boundaries.

The analysis of (4.4) will yield an estimate of $|\hat{\mathbf{w}}|$ for $x<-\alpha$. Note that the fringe function $\bar{\lambda}$ and consequently also $\hat{F}$ is nonzero for $x \in[-\alpha, 0], 0<\alpha<\infty$, where $\alpha$ is the length of the fringe region.

The rest of this paper will deal with problems (4.3) and (4.4). The analysis of (4.3) will indicate if it is possible to force the solution toward zero inside the fringe region. The analysis of (4.4) will indicate whether that procedure leads to significant upstream influence from the fringe region. The relevance of the model problems (4.3) and (4.4) is tested in section 6 when the theoretical and computational results are compared.

For later reference, the definitions of the Laplace and Fourier transforms and their corresponding inverses are given below:

$$
\begin{aligned}
& \tilde{\phi}(y, s)=\int_{0}^{\infty} e^{-s t} \phi(y, t) d t, \quad \phi(y, t)=\frac{1}{2 \pi i} \int_{\eta-i \infty}^{\eta+i \infty} e^{s t} \tilde{\phi}(y, s) d s \\
& \hat{\phi}(\omega, t)=\frac{1}{2 \pi} \int_{-\infty}^{\infty} e^{-i \omega y} \phi(y, t) d y, \quad \phi(y, t)=\int_{-\infty}^{\infty} e^{i \omega y} \hat{\phi}(\omega, t) d \omega .
\end{aligned}
$$

5. Analysis of the constant coefficient problems. The Laplace transformed version of $\hat{\mathbf{w}}_{t}^{1}=\bar{P} \hat{\mathbf{w}}-\bar{\lambda} \hat{\mathbf{w}}^{1}+\hat{F}, \hat{\nabla} \cdot \hat{\mathbf{w}}^{1}=0$ can be written as a first-order system of 
ordinary differential equations

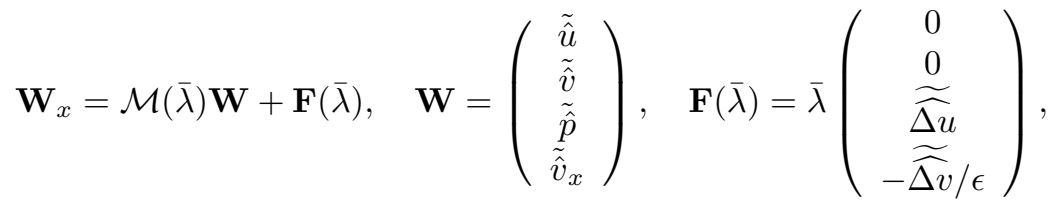

$$
\begin{aligned}
& \mathcal{M}(\bar{\lambda})=\left(\begin{array}{cccc}
0 & -i \omega & 0 & 0 \\
0 & 0 & 0 & 1 \\
-\left(s+\bar{\lambda}+i \omega \bar{v}+\epsilon \omega^{2}\right) & i \omega \bar{u} & 0 & -i \omega \epsilon \\
0 & \left(s+\bar{\lambda}+i \omega \bar{v}+\epsilon \omega^{2}\right) / \epsilon & i \omega / \epsilon & \bar{u} / \epsilon
\end{array}\right),
\end{aligned}
$$

with the solution

$$
\mathbf{W}=\mathbf{W}_{h}+\mathbf{W}_{p}, \quad \mathbf{W}_{h}=e^{x \mathcal{M}} \mathbf{W}_{0}, \quad \mathbf{W}_{p}=\int_{0}^{x} e^{(x-\xi) \mathcal{M}} \mathbf{F} d \xi
$$

Note that in the inflow case $\mathbf{F}=\mathbf{F}(0)=0$ and in the outflow case $\mathcal{M}=\mathcal{M}(0)$. The eigenvalues of $\mathcal{M}$ are

$$
\kappa_{1}=+|\omega|, \quad \kappa_{2}=-|\omega|, \quad \kappa_{3}=+|\omega|+\Delta_{3}, \quad \kappa_{4}=-|\omega|-\Delta_{4},
$$

where

$$
\begin{aligned}
& \Delta_{3}=+\frac{\bar{u}-2 \epsilon|\omega|}{2 \epsilon}\left(1+\vartheta \sqrt{1+\frac{4 \epsilon \sigma_{1}}{(\bar{u}-2 \epsilon|\omega|)^{2}}}\right), \\
& \Delta_{4}=-\frac{\bar{u}+2 \epsilon|\omega|}{2 \epsilon}\left(1-\sqrt{1+\frac{4 \epsilon \sigma_{2}}{(\bar{u}+2 \epsilon|\omega|)^{2}}}\right),
\end{aligned}
$$

and

$$
\vartheta=\operatorname{sign}(\bar{u}-2 \epsilon|\omega|), \quad \sigma_{1}=s+\bar{\lambda}+\bar{u}|\omega|+i \omega \bar{v}, \quad \sigma_{2}=s+\bar{\lambda}-\bar{u}|\omega|+i \omega \bar{v} .
$$

For $\operatorname{Re}(s) \geq 0$ there is one double root, i.e., $\lim \kappa_{4 \sigma_{2} \rightarrow 0}=-|\omega|=\kappa_{2}$.

The relation between the solution for distinct roots and the solution with one double root is discussed in Appendix A. It is shown that the solution (5.1) can be written

$$
\begin{aligned}
\mathbf{W}(x)= & {\left[x_{1}+\int_{0}^{x} \bar{\lambda} a e^{-|\omega| \xi} d \xi\right] e^{|\omega| x} \psi_{1}+\left[x_{2}+\int_{0}^{x} \bar{\lambda} b e^{|\omega| \xi} d \xi\right] e^{-|\omega| x} \psi_{2} } \\
& +\left[x_{3}+\int_{0}^{x} \bar{\lambda} c e^{-\kappa_{3} \xi} d \xi\right] e^{\kappa_{3} x} \psi_{3}+\left[x_{4}+\int_{0}^{x} \bar{\lambda} d e^{-\kappa_{4} \xi} d \xi\right] e^{\kappa_{4} x} \psi_{4}
\end{aligned}
$$

where

$$
\psi_{1}=\left(\begin{array}{c}
1 \\
i \frac{|\omega|}{\omega} \\
-\frac{\sigma_{1}}{\omega \mid} \\
i \omega
\end{array}\right), \quad \psi_{3}=\left(\begin{array}{c}
1 \\
i \frac{\kappa_{3}}{\omega} \\
0 \\
i \frac{\kappa_{3}^{2}}{\omega}
\end{array}\right), \quad \psi_{2}=\left(\begin{array}{c}
1 \\
-i \frac{|\omega|}{\omega} \\
\frac{\sigma_{2}}{|\omega|} \\
i \omega
\end{array}\right), \quad \psi_{4}=\left(\begin{array}{c}
1 \\
i \frac{\kappa_{4}}{\omega} \\
0 \\
i \frac{\kappa_{4}^{2}}{\omega}
\end{array}\right)
$$

In the inflow problem $(\mathbf{F}=0), a=b=c=d=0$, while for the outflow problem $(\mathbf{F} \neq 0), a, b, c, d$ are given in (A.20), (A.21), and (A.22). The constants $x_{1}, x_{2}, x_{3}, x_{4}$ in (5.6) will be determined by the boundary conditions. 
5.1. Data considerations. Boundary data $g=\left(u_{0}, v_{0}\right)^{T}$ and forcing function $F=\bar{\lambda} \Delta \mathbf{u}^{1}$ (see (4.2), (4.3), (4.4)) which satisfy

$$
\begin{gathered}
g(y, 0)=0, \quad \int_{-\infty}^{+\infty} u_{0}(y, t) d y=0, \quad(g)_{|y|>\delta}=0, \quad\left(\Delta \mathbf{u}^{1}\right)_{|y|>\delta}=0, \\
\left|\frac{\partial^{r+j} g}{\partial t^{j} \partial y^{r}}\right|_{|y| \leq \delta} \leq \text { const. }, \quad\left|\frac{\partial^{r} \Delta \mathbf{u}^{1}}{\partial y^{r}}\right|_{|y| \leq \delta} \leq \text { const., } \quad j=0,1, \quad r=0,1,2 \ldots,
\end{gathered}
$$

are considered. The first condition in (5.8) is required to get compatibility at $t=0$, the second condition guarantees that no additional momentum is added by the fringe region technique, and the next two conditions state that the data vanish for $|y|>\delta$. The assumption that $g, g_{t}, \Delta \mathbf{u}^{1}$ are sufficiently smooth for $|y| \leq \delta$ is formulated in (5.9).

In Appendix B it is shown that (5.8) and (5.9) lead to

$$
\begin{gathered}
\hat{g}_{t}(\omega, t)=\mathcal{L}^{-1} s \tilde{\hat{g}}(\omega, s), \quad\left|\frac{\partial^{j} \hat{u}_{0}(\omega, t)}{\partial t^{j}}\right| \leq \mathcal{C}_{0} \max _{-\delta \leq y \leq+\delta}\left|\frac{\partial^{j} u_{0}(y, t)}{\partial t^{j}}\right||\omega|, \\
\left|\omega^{r} \frac{\partial^{j} \hat{g}}{\partial t^{j}}\right| \leq \mathcal{C}_{1}, \quad\left|\omega^{r} \widehat{\Delta \mathbf{u}}^{1}\right| \leq \mathcal{C}_{2}
\end{gathered}
$$

where $j=0,1$ and $\mathcal{C}_{0}, \mathcal{C}_{1}, \mathcal{C}_{2}$ are constants.

5.2. The inflow problem. The constants $x_{1}, x_{2}, x_{3}, x_{4}$ in (5.6) for the inflow problem are determined in Appendix D. The exact solution to (4.3) in Laplace space becomes

$$
\mathbf{W}(x)=\frac{\kappa_{4} \tilde{\hat{u}}_{0}+i \omega \tilde{\hat{v}}_{0}}{\kappa_{4}+|\omega|} \psi_{2} e^{-|\omega| x}+\frac{|\omega| \tilde{\hat{u}}_{0}-i \omega \tilde{\hat{v}}_{0}}{\kappa_{4}+|\omega|} \psi_{4} e^{\kappa_{4} x} .
$$

Estimates of $\mathbf{W}$ and the proof of the following theorem are given in Appendix D.

THEOREM 5.1. The solution of the inflow problem (4.3) satisfies

$$
\begin{aligned}
& \int_{0}^{\infty}|\hat{u}(x, \omega, t)|^{2} e^{-2 \eta t} d t \leq \mathcal{C}_{1} e^{-2 \theta x} \int_{0}^{\infty}\left\{(1+|\omega|)^{2}\left|\hat{u}_{0}\right|^{2}+|\omega|^{2}\left|\hat{v}_{0}\right|^{2}\right\} e^{-2 \eta t} d t \\
& \int_{0}^{\infty}|\hat{v}(x, \omega, t)|^{2} e^{-2 \eta t} d t \leq \mathcal{C}_{2} e^{-2 \theta x} \int_{0}^{\infty}\left\{(1+|\omega|)^{2}\left|\hat{u}_{0}\right|^{2}+|\omega|^{2}\left|\hat{v}_{0}\right|^{2}\right\} e^{-2 \eta t} d t
\end{aligned}
$$

$$
\begin{aligned}
& \int_{0}^{\infty}|\hat{p}(x, \omega, t)|^{2} e^{-2 \eta t} d t \\
& \quad \leq \mathcal{C}_{3} e^{-2|\omega| x} \int_{0}^{\infty}\left\{\left(\frac{1+|\omega|}{|\omega|}\right)^{2}\left(\left|\left(\hat{u}_{0}\right)_{t}\right|^{2}+(1+|\omega|)^{2}\left|\hat{u}_{0}\right|^{2}\right)\right\} e^{-2 \eta t} d t
\end{aligned}
$$

$$
\begin{aligned}
+\mathcal{C}_{3} e^{-2|\omega| x} \int_{0}^{\infty}\left\{\left|\left(\hat{v}_{0}\right)_{t}\right|^{2}+\right. & \left.(1+|\omega|)^{2}\left|\hat{v}_{0}\right|^{2}\right\} e^{-2 \eta t} d t \\
\int_{0}^{\infty}\left|\left(\widehat{p_{y}}\right)(x, \omega, t)\right|^{2} e^{-2 \eta t} d t & =\int_{0}^{\infty}\left|\left(\widehat{p_{x}}\right)(x, \omega, t)\right|^{2} e^{-2 \eta t} d t \\
& \leq|\omega|^{2} \int_{0}^{\infty}|\hat{p}(x, \omega, t)|^{2} e^{-2 \eta t} d t
\end{aligned}
$$




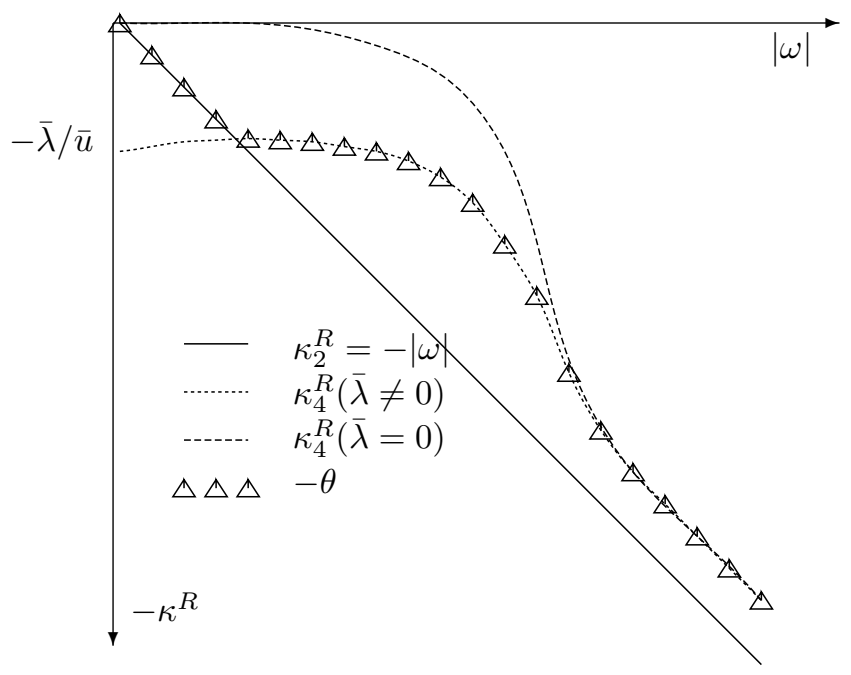

FIG. 5.1. Schematic view of $\operatorname{Re}\left(\kappa_{2}\right)=\kappa_{2}^{R}, \operatorname{Re}\left(\kappa_{4}\right)=\kappa_{4}^{R}$, and $\theta$ as a function of $|\omega|$.

where

$$
\theta=\min _{\xi}\left(|\omega|, \operatorname{Re}\left(\kappa_{4}\right)\right)=\left\{\begin{array}{ll}
|\omega|, & |\omega| \leq(\bar{\lambda}+\eta) / \bar{u} \\
|\omega|+\min _{\xi} \operatorname{Re}\left(\Delta_{4}\right), & |\omega|>(\bar{\lambda}+\eta) / \bar{u}
\end{array}\right\} .
$$

In (5.13)-(5.17), $0 \leq \eta=\operatorname{Re}(s), \xi=\operatorname{Im}(s), x>0, \mathcal{C}_{1}, \mathcal{C}_{2}, \mathcal{C}_{3}$ are constants, and $\Delta_{4}$ is defined in (5.4).

We stress the important role of $\bar{\lambda}$. Let $s=0, \bar{v}=0, \bar{\lambda}=0$; then the function $\theta$ in (5.13)-(5.15) becomes $\theta \approx-\epsilon|\omega|^{2} / \bar{u}$ for $|\epsilon \omega| \ll 1$. This leads to a very slow exponential decay with respect to $x$ of the low frequency modes in the solution. With $\bar{\lambda} \neq 0$ one obtains a significant improvement for small $|\omega|$ (see Figure 5.1) since $\operatorname{Re}\left(\kappa_{4}(\bar{\lambda} \neq 0)\right) \approx-\bar{\lambda} / \bar{u}$ for small $|\omega|$. Note that the presence of $\bar{\lambda}$ has no influence on the decay of the error in the pressure.

The exponential decay of the solution depends on $|\omega|$. Note that $\theta=|\omega|$ for small $\omega$ if $\bar{\lambda} \neq 0$. Obviously, $|\omega| \rightarrow 0$ is a crucial case. The solution seems to be of order 1 in the velocities and pressure gradients and of order $|\omega|^{-1}$ in the pressure. However, (5.13)-(5.15) and (5.10)-(5.11) evaluated for small $\omega$ lead to

$$
\begin{aligned}
\int_{0}^{\infty}|\hat{u}(x, \omega, t)|^{2} e^{-2 \eta t} d t \leq & \mathcal{C}_{4}\left(|\omega| e^{-|\omega| x}\right)^{2} \\
& \times \int_{0}^{\infty}\left\{\left|u_{0}(y, t)\right|_{m}^{2}+\left|v_{0}(y, t)\right|_{m}^{2}\right\} e^{-2 \eta t} d t \\
\int_{0}^{\infty}|\hat{v}(x, \omega, t)|^{2} e^{-2 \eta t} d t \leq & \mathcal{C}_{5}\left(|\omega| e^{-|\omega| x}\right)^{2}
\end{aligned}
$$

$$
\times \int_{0}^{\infty}\left\{\left|u_{0}(y, t)\right|_{m}^{2}+\left|v_{0}(y, t)\right|_{m}^{2}\right\} e^{-2 \eta t} d t
$$

$$
\begin{aligned}
\int_{0}^{\infty}|\hat{p}(x, \omega, t)|^{2} e^{-2 \eta t} d t \leq & \mathcal{C}_{6}\left(e^{-|\omega| x}\right)^{2} \int_{0}^{\infty}\left\{\left|u_{0}(y, t)\right|_{m}^{2}+\left|v_{0}(y, t)\right|_{m}^{2}\right\} e^{-2 \eta t} d t \\
& +\mathcal{C}_{6}\left(e^{-|\omega| x}\right)^{2} \int_{0}^{\infty}\left\{\left|\left(u_{0}\right)_{t}(y, t)\right|_{m}^{2}+\left|\left(v_{0}\right)_{t}(y, t)\right|_{m}^{2}\right\} e^{-2 \eta t} d t,
\end{aligned}
$$


where $\mathcal{C}_{4}, \mathcal{C}_{5}, \mathcal{C}_{6}$ are constants and

$$
\begin{aligned}
& \left|\frac{\partial^{j} u_{0}(y, t)}{\partial t^{j}}\right|_{m}=\max _{-\delta \leq y \leq+\delta}\left|\frac{\partial^{j} u_{0}(y, t)}{\partial t^{j}}\right|, \\
& \left|\frac{\partial^{j} v_{0}(y, t)}{\partial t^{j}}\right|_{m}=\max _{-\delta \leq y \leq+\delta}\left|\frac{\partial^{j} v_{0}(y, t)}{\partial t^{j}}\right|, \quad j=0,1 .
\end{aligned}
$$

We can summarize the result in the following way. For large $|\omega|$ there is a fast exponential decay of the solution in the fringe region (estimates (5.13)-(5.15)). Note that the estimate (5.10)-(5.11) with $r$ sufficiently large means that $\hat{g}, \hat{g}_{t}$ and hence also $\hat{u}, \hat{v}, \hat{p}, \widehat{p_{x}}, \widehat{p_{y}}$ decay fast as $|\omega| \rightarrow \infty$. For small $|\omega|$, the exponential decay is small but, on the other hand, the solution is of order $|\omega|$ in the velocities and pressure gradients while it is of order 1 in the pressure (estimates (5.18)-(5.20)). The primary importance of the fringe function $\bar{\lambda}$ is to damp out the deviation associated with small $|\omega|$.

5.3. The outflow problem. The constants $x_{1}, x_{2}, x_{3}, x_{4}$ in (5.6) for the outflow problem are determined in Appendix E. The exact solution to (4.4) in Laplace space becomes $\mathbf{W}(x)=\mathbf{W}_{h}(x)+\mathbf{W}_{p}(x)$, where

$$
\mathbf{W}_{h}(x)=\frac{\kappa_{3} \tilde{\hat{u}}_{0}+i \omega \tilde{\hat{v}}_{0}}{\kappa_{3}-|\omega|} \psi_{1} e^{|\omega| x}-\frac{|\omega| \tilde{\hat{u}}_{0}+i \omega \tilde{\hat{v}}_{0}}{\kappa_{3}-|\omega|} \psi_{3} e^{\kappa_{3} x}
$$$$
\mathbf{W}_{p}(x)=\left[\int_{0}^{-\alpha} \bar{\lambda} \frac{\left(\kappa_{3}+|\omega|\right) b e^{|\omega| \xi}+\left(\kappa_{3}-\kappa_{4}\right) d e^{-\kappa_{4} \xi}}{\kappa_{3}-|\omega|} d \xi+\int_{0}^{x} \bar{\lambda} a e^{-|\omega| \xi} d \xi\right] \psi_{1} e^{|\omega| x}
$$$$
-\left[\int_{x}^{-\alpha} \bar{\lambda} b e^{|\omega| \xi} d \xi\right] \psi_{2} e^{-|\omega| x}
$$$$
+\left[\int_{0}^{-\alpha} \bar{\lambda} \frac{-2|\omega| b e^{|\omega| \xi}+\left(\kappa_{4}-|\omega|\right) d e^{-\kappa_{4} \xi}}{\kappa_{3}-|\omega|} d \xi+\int_{0}^{x} \bar{\lambda} c e^{-\kappa_{3} \xi} d \xi\right] \psi_{3} e^{\kappa_{3} x}
$$$$
-\left[\int_{x}^{-\alpha} \bar{\lambda} d e^{-\kappa_{4} \xi} d \xi\right] \psi_{4} e^{\kappa_{4} x} \text {. }
$$

Estimates of $\mathbf{W}$ and the proof of the following theorem are given in Appendix E.

THEOREM 5.2. Consider the outflow problem (4.4). The particular solution satisfies

$$
\begin{aligned}
\int_{0}^{\infty}\left|\hat{u}_{p}(x, \omega, t)\right|^{2} e^{-2 \eta t} d t \leq & \mathcal{C}_{1}\left(\bar{\lambda} \alpha e^{|\omega|(x+\alpha)}\right)^{2}\left(\frac{|\omega|}{1+|\omega|^{2}}\right)^{2} \\
& \times \int_{0}^{\infty}\left\{|\widehat{\Delta u}(x, \omega, t)|_{m}^{2}+|\widehat{\Delta v}(x, \omega, t)|_{m}^{2}\right\} e^{-2 \eta t} d t \\
\int_{0}^{\infty}\left|\hat{v}_{p}(x, \omega, t)\right|^{2} e^{-2 \eta t} d t \leq & \mathcal{C}_{2}\left(\bar{\lambda} \alpha e^{|\omega|(x+\alpha)}\right)^{2}\left(\frac{|\omega|}{1+|\omega|^{2}}\right)^{2}
\end{aligned}
$$

$$
\times \int_{0}^{\infty}\left\{|\widehat{\Delta u}(x, \omega, t)|_{m}^{2}+|\widehat{\Delta v}(x, \omega, t)|_{m}^{2}\right\} e^{-2 \eta t} d t
$$

$$
\int_{0}^{\infty}\left|\hat{p}_{p}(x, \omega, t)\right|^{2} e^{-2 \eta t} d t \leq \mathcal{C}_{3}\left(\bar{\lambda} \alpha e^{|\omega|(x+\alpha)}\right)^{2}\left(\frac{1}{1+|\omega|}\right)^{2}
$$

$$
\times \int_{0}^{\infty}\left\{|\widehat{\Delta u}(x, \omega, t)|_{m}^{2}+|\widehat{\Delta v}(x, \omega, t)|_{m}^{2}\right\} e^{-2 \eta t} d t,
$$


where

$$
|\widehat{\Delta u}(x, \omega, t)|_{m}=\max _{-\alpha \leq x \leq 0}|\widehat{\Delta u}(x, \omega, t)|, \quad|\widehat{\Delta v}(x, \omega, t)|_{m}=\max _{-\alpha \leq x \leq 0}|\widehat{\Delta v}(x, \omega, t)| .
$$

The homogeneous solution satisfies

$$
\begin{aligned}
& \int_{0}^{\infty}\left|\hat{u}_{h}(x, \omega, t)\right|^{2} e^{-2 \eta t} d t \leq \mathcal{C}_{4}\left(e^{|\omega|(x+\alpha)}\right)^{2} \\
& \times \int_{0}^{\infty}\left\{(1+\epsilon|\omega|)^{2}\left|\hat{u}_{0}(\omega, t)\right|^{2}+\epsilon^{2}|\omega|^{2}\left|\hat{v}_{0}(\omega, t)\right|^{2}\right\} e^{-2 \eta t} d t \\
& \int_{0}^{\infty}\left|\hat{v}_{h}(x, \omega, t)\right|^{2} e^{-2 \eta t} d t \leq \mathcal{C}_{5}\left(e^{|\omega|(x+\alpha)}\right)^{2} \\
& \times \int_{0}^{\infty}\left\{(1+\epsilon|\omega|)^{2}\left|\hat{u}_{0}(\omega, t)\right|^{2}+\epsilon^{2}|\omega|^{2}\left|\hat{v}_{0}(\omega, t)\right|^{2}\right\} e^{-2 \eta t} d t \\
& \int_{0}^{\infty}\left|\hat{p}_{h}(x, \omega, t)\right|^{2} e^{-2 \eta t} d t \\
& \leq \mathcal{C}_{6}\left(e^{|\omega|(x+\alpha)}\right)^{2} \int_{0}^{\infty}\left\{(1+\epsilon|\omega|)^{2}\left|\hat{u}_{0}(\omega, t)\right|^{2}+\epsilon^{2}|\omega|^{2}\left|\hat{v}_{0}(\omega, t)\right|^{2}\right\} e^{-2 \eta t} d t \\
& +\mathcal{C}_{6}\left(e^{|\omega|(x+\alpha)}\right)^{2} \int_{0}^{\infty}\left\{\left(\frac{1+\epsilon|\omega|}{|\omega|}\right)^{2}\left|\left(\hat{u}_{0}\right)_{t}(\omega, t)\right|^{2}+\epsilon^{2}\left|\left(\hat{v}_{0}\right)_{t}(\omega, t)\right|^{2}\right\} e^{-2 \eta t} d t .
\end{aligned}
$$

The estimate of the pressure gradient is

$$
\begin{aligned}
\int_{0}^{\infty}\left|\left(\widehat{p_{y}}\right)(x, \omega, t)\right|^{2} e^{-2 \eta t} d t & =\int_{0}^{\infty}\left|\left(\widehat{p_{x}}\right)(x, \omega, t)\right|^{2} e^{-2 \eta t} d t \\
& \leq|\omega|^{2} \int_{0}^{\infty}|\hat{p}(x, \omega, t)|^{2} e^{-2 \eta t} d t
\end{aligned}
$$

where $\hat{p}=\hat{p}_{h}+\hat{p}_{p} . \quad$ In $(5.25)-(5.31), x<-\alpha, \eta \geq 0$, and $\mathcal{C}_{1}, \mathcal{C}_{2}, \mathcal{C}_{3}, \mathcal{C}_{4}, \mathcal{C}_{5}, \mathcal{C}_{6}$ are constants.

The exponential decay of the solution outside the fringe region, $x<-\alpha$, depends on $|\omega|$. Just as in the inflow problem, $|\omega| \rightarrow 0$ is a crucial case. For small $|\omega|$, the particular solution is of order $|\omega|$ in the velocities and pressure gradients while it is of order 1 in the pressure. Worse is that the homogeneous solution seems to be of order 1 in the velocities and pressure gradients and of order $|\omega|^{-1}$ in the pressure. However, (5.28)-(5.30), (5.10)-(5.11), and (5.21)-(5.22) lead to

$$
\begin{aligned}
\int_{0}^{\infty}\left|\hat{u}_{h}(x, \omega, t)\right|^{2} e^{-2 \eta t} d t \leq & \mathcal{C}_{7}\left(|\omega| e^{|\omega|(x+\alpha)}\right)^{2} \\
& \times \int_{0}^{\infty}\left\{\left|u_{0}(y, t)\right|_{m}^{2}+\epsilon^{2}\left|v_{0}(y, t)\right|_{m}^{2}\right\} e^{-2 \eta t} d t \\
\int_{0}^{\infty}\left|\hat{v}_{h}(x, \omega, t)\right|^{2} e^{-2 \eta t} d t \leq & \mathcal{C}_{8}\left(|\omega| e^{|\omega|(x+\alpha)}\right)^{2} \\
& \times \int_{0}^{\infty}\left\{\left|u_{0}(y, t)\right|_{m}^{2}+\epsilon^{2}\left|v_{0}(y, t)\right|_{m}^{2}\right\} e^{-2 \eta t} d t
\end{aligned}
$$




$$
\begin{aligned}
\int_{0}^{\infty}\left|\hat{p}_{h}(x, \omega, t)\right|^{2} e^{-2 \eta t} d t \\
\leq \leq \\
\quad \mathcal{C}_{9}\left(|\omega| e^{|\omega|(x+\alpha)}\right)^{2} \int_{0}^{\infty}\left\{\left|u_{0}(y, t)\right|_{m}^{2}+\epsilon^{2}\left|v_{0}(y, t)\right|_{m}^{2}\right\} e^{-2 \eta t} d t \\
\quad+\mathcal{C}_{9}\left(e^{|\omega|(x+\alpha)}\right)^{2} \int_{0}^{\infty}\left\{\left|\left(u_{0}\right)_{t}(y, t)\right|_{m}^{2}+\epsilon^{2}\left|\left(v_{0}\right)_{t}(y, t)\right|_{m}^{2}\right\} e^{-2 \eta t} d t
\end{aligned}
$$

where $\mathcal{C}_{7}, \mathcal{C}_{8}, \mathcal{C}_{9}$ are constants.

We can summarize the result in the following way. Large $|\omega|$ leads to a fast exponential decay of the solution outside the fringe region (estimates (5.25)-(5.30)). The estimate (5.10)-(5.11) with $r$ sufficiently large means that $\hat{g}, \hat{g}_{t}, \widehat{\Delta \mathbf{u}}^{1}$ and hence also $\hat{u}, \hat{v}, \hat{p}, \widehat{p_{x}}, \widehat{p_{y}}$ decay fast as $|\omega| \rightarrow \infty$. For small $|\omega|$, the exponential decay is small but on the other hand, the solution is of order $|\omega|$ in the velocities and pressure gradients, while it is of order 1 in the pressure (estimates (5.25)-(5.27), (5.32)-(5.34)). Note that the presence of $\bar{\lambda}$ increases the upstream influence from the fringe region; see (5.25)-(5.27). However, $\bar{\lambda}$ is multiplied with $|\omega|$ in (5.25), (5.26). This means that for $|\omega|$ small, the effect on the velocities and pressure gradients is small. For large $|\omega|$, the value of $\bar{\lambda}$ is irrelevant due to the fast exponential decay of the solution.

6. Numerical experiments. The upstream influence of the fringe region as well as the ability to force the solution to the specified inflow values will be studied computationally and compared with the previous theoretical predictions.

6.1. The numerical method and the fringe region technique. The numerical algorithm in the simulation code uses Fourier series expansions in the horizontal directions, Chebyshev series in the normal direction, and pseudospectral treatment of the nonlinear terms. The code, developed by Lundbladh, Henningson, and Johansson [19], has been thoroughly checked and used on a variety of supercomputers. It was designed to compute temporally evolving flows. Recently, free-stream boundary conditions and the fringe region technique were added to be able to compute spatially developing boundary layer flows; see [8], [9].

The fringe function (see Figure 2.2) in the code has the following form:

$$
\lambda(x)=\bar{\lambda}\left[S\left(\frac{x-x_{\text {start }}}{\Delta_{\text {rise }}}\right)-S\left(\frac{x-x_{\text {end }}}{\Delta_{\text {fall }}}+1\right)\right],
$$

where $\bar{\lambda}$ is the maximum strength of the damping and $x_{\text {start }}-x_{\text {end }}=\alpha$ the spatial extent of the region where the damping function is nonzero. $\Delta_{\text {rise }}, \Delta_{\text {fall }}$ are the rise and fall distance of the damping function, respectively, and $S(x)$ is a smooth step function rising from zero for negative $x$ to 1 for $x \geq 1$. We have used

$$
S(x)= \begin{cases}0, & x \leq 0 \\ 1 /\left[1+\exp \left(\frac{1}{x-1}+\frac{1}{x}\right)\right], & 0<x<1, \\ 1, & x \geq 1\end{cases}
$$

which has the advantage of having continuous derivatives of all orders.

The function ú in (2.3) is used to prescribe the inflow conditions. Ideally, it should be an exact solution to the Navier-Stokes equations. In the code, $\dot{\mathbf{u}}^{1}=\mathbf{u}_{B L}^{1}=$ $\left(u_{B L}, v_{B L}\right)^{T}$ is used, where

$$
u_{B L}=U_{B}(x, y)+\left[U_{B}(x-L, y)-U_{B}(x, y)\right] S\left(\frac{x-x_{m i x}}{\Delta_{m i x}}\right)
$$


TABLE 6.1

Parameters used in the first set of numerical simulations. $y_{\max }$ is the height of the computational box. The grid corresponds to the number of modes used in the streamwise and normal directions, respectively. In addition we have used $L=600, x_{\text {start }}=300, x_{\text {end }}=600, \Delta_{\text {rise }}=100$, $\Delta_{\text {fall }}=40, x_{\text {mix }}=300$, and $\Delta_{\text {mix }}=80$ for these simulations.

\begin{tabular}{|c|r|c|l|c|}
\hline Run & \multicolumn{1}{|c|}{$R_{\delta_{*}}$} & $y_{\max }$ & \multicolumn{1}{|c|}{$\lambda$} & grid \\
\hline $\mathrm{A}$ & 2400 & 10 & 0.01 & $384 \times 49$ \\
$\mathrm{~B}$ & 2400 & 10 & 0.025 & $384 \times 49$ \\
$\mathrm{C}$ & 2400 & 10 & 0.05 & $384 \times 49$ \\
$\mathrm{D}$ & 2400 & 10 & 0.1 & $384 \times 49$ \\
$\mathrm{E}$ & 2400 & 10 & 0.25 & $384 \times 49$ \\
$\mathrm{~F}$ & 2400 & 10 & 0.5 & $384 \times 49$ \\
$\mathrm{G}$ & 2400 & 10 & 1.0 & $384 \times 49$ \\
$\mathrm{H}$ & 2400 & 10 & 4.0 & $384 \times 49$ \\
$\mathrm{I}$ & 1600 & 10 & 1.0 & $384 \times 49$ \\
$\mathrm{~J}$ & 800 & 10 & 1.0 & $384 \times 49$ \\
$\mathrm{~K}$ & 2400 & 20 & 1.0 & $384 \times 97$ \\
\hline
\end{tabular}

TABLE 6.2

Parameters used in the second set of numerical simulations. In addition we have used $R_{\delta_{*}}=$ $2400, y_{\max }=10, \bar{\lambda}=1, x_{m i x}=300, \Delta_{m i x}=25$, and a grid of $384 \times 49$ for these simulations.

\begin{tabular}{|c|c|c|c|c|c|}
\hline Run & $\mathrm{L}$ & $x_{\text {start }}$ & $x_{\text {end }}$ & $\Delta_{\text {rise }}$ & $\Delta_{\text {fall }}$ \\
\hline $\mathrm{L}$ & 600 & 300 & 600 & 70 & 15 \\
$\mathrm{M}$ & 550 & 300 & 550 & 70 & 15 \\
$\mathrm{~N}$ & 500 & 300 & 500 & 70 & 15 \\
$\mathrm{O}$ & 450 & 300 & 450 & 70 & 15 \\
$\mathrm{P}$ & 400 & 300 & 400 & 70 & 15 \\
\hline
\end{tabular}

and $v_{B L}$ is calculated from the continuity equation. $U_{B}(x, y)$ is typically a solution of the boundary layer equations, for zero pressure gradient it is the Blasius solution. $L$ is the streamwise length of the periodic simulation box. The parameters $x_{m i x}$ and $\Delta_{m i x}$ are chosen so that the prescribed flow smoothly changes from the outflow velocity to the inflow velocity within the fringe region. This choice of $\mathbf{u}$ ensures that the decrease in boundary layer thickness is confined to the fringe region.

In the simulation code, all quantities are normalized by the displacement thickness at the inflow boundary $\delta_{*}$ and the free-stream velocity $U_{\infty}$. For the Blasius boundary layer this means that $R_{\delta_{*}}=1.708 \epsilon^{-1 / 2}$, where $R_{\delta_{*}}$ is the Reynolds number based on the displacement thickness and $\epsilon$ is the previously defined inverse of the Reynolds number based on the distance from the leading edge. In the comparison below of the simulation results with the theoretical predictions, all quantities are nondimensionalized with $\delta_{*}$ and $U_{\infty}$.

First, results from 11 simulations will be presented. The parameters used in the simulations are listed in Table 6.1. Other simulations have been done in order to check that the results are converged. Note that the fringe region parameters in this study do not represent the most computationally efficient ones. Instead, they are chosen to facilitate a comparison with the theoretical results from the analysis above. In particular, the fringe region used in most of this study is substantially longer than the one usually used in practice.

A second set of simulations, where the length of the fringe region is varied, is performed in order to show that shorter lengths also give adequate damping. The parameters chosen for those simulations are found in Table 6.2.

An investigation of how well the solution $\mathbf{v}^{1}=(u, v)^{T}$ obtained with the fringe region technique approximates an exact solution to the Navier-Stokes equations will 


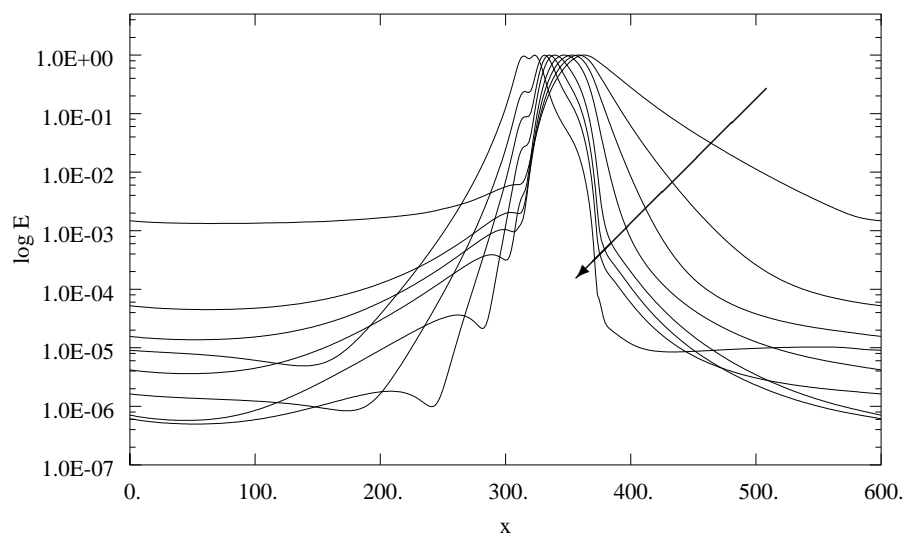

FIG. 6.1. Numerical experiments with varying $\bar{\lambda}$ showing the energy of the error vs. the streamwise distance. Results from simulations $A$ through $H$ are shown in the direction of the arrow. The maxima of all curves have been normalized to 1.

be made below. The energy of the error $(E)$ is defined in the following way:

$$
E=\int_{0}^{y_{\max }}\left[\left(u-u_{B L}\right)^{2}+\left(v-v_{B L}\right)^{2}\right] d y .
$$

Recall that $\mathbf{u}_{B L}^{1}$ coincides with the Blasius solution in the physical part of the computational domain $(S=0)$ as well as in the latter part of the fringe region $(S=1)$; see (6.2). Thus it can be used to estimate both the upstream influence of the fringe region and the ability to force the flow toward the correct solution.

Due to the lack of an exact solution to the Navier-Stokes equations $\left(\mathbf{u}_{B L}^{1}\right.$ is an approximation of that solution), we cannot expect the error to be exactly zero in the physical domain. However, the difference between the Navier-Stokes solution and the boundary layer solution $\mathbf{u}_{B L}^{1}$ decreases with increasing Reynolds number. At the Reynolds numbers used in this investigation (see Table 6.1) the difference is small enough to make $E$ in (6.3) a relevant measure of the error in the fringe region technique.

In the simulations $\mathrm{F}-\mathrm{H}$ in Table 6.1 , values of $E$ in the physical part of the domain below $10^{-9}$ were obtained. This exemplifies the typical accuracy of this technique and implies that the Blasius solution was approximated to about five significant digits.

6.2. Theoretical and computational results: The inflow problem. Recall that the fringe region starts at the outflow boundary $(x=300)$ and ends at the inflow boundary $(x=600)$. The error $E$ in $(6.3)$ as a function of the streamwise distance $x$ is shown in Figure 6.1. For small $\bar{\lambda}$ (the top curves in the figure), the error decays slowly toward the end of the fringe region. When $\bar{\lambda}$ increases, the error decays faster in the region $350<x<450$. For larger $x$, the decay slows down. Note that the large errors at the inflow boundary for small $\bar{\lambda}$ leads to large errors in the physical region of the computational domain, $0<x<300$.

Figure 5.1, which summarizes the most important theoretical result in Theorem 5.1 , shows that errors with small normal scales (large $\omega$ ) decay fast with a decay rate proportional to $\bar{\lambda}$, whereas errors with large normal scales (small $\omega$ ) decay slower with a rate proportional to $\omega$. Thus, for large $\bar{\lambda}$ one would first expect to see a fast decay associated with small normal scales. Further downstream a slower decay associated 


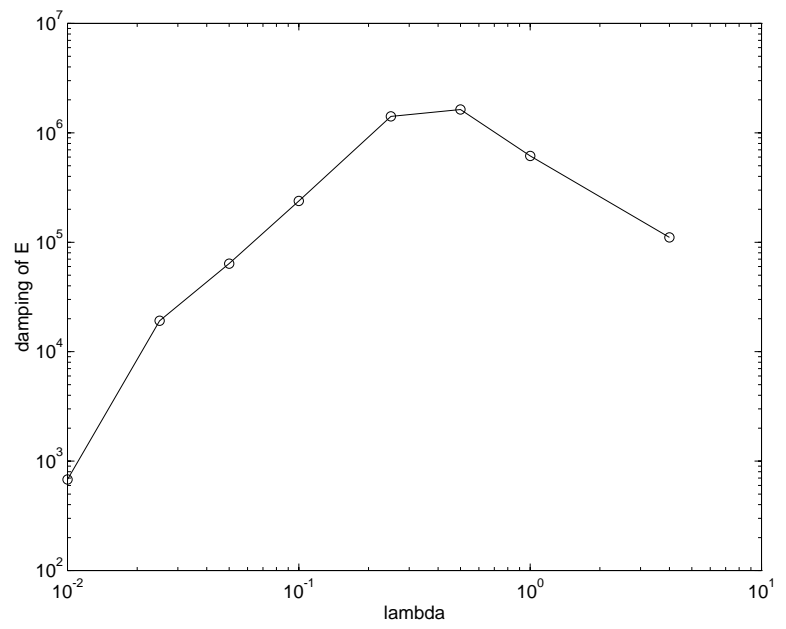

FIG. 6.2. Damping of the fringe region as a function of $\bar{\lambda}$, simulations $A$ through $H$.

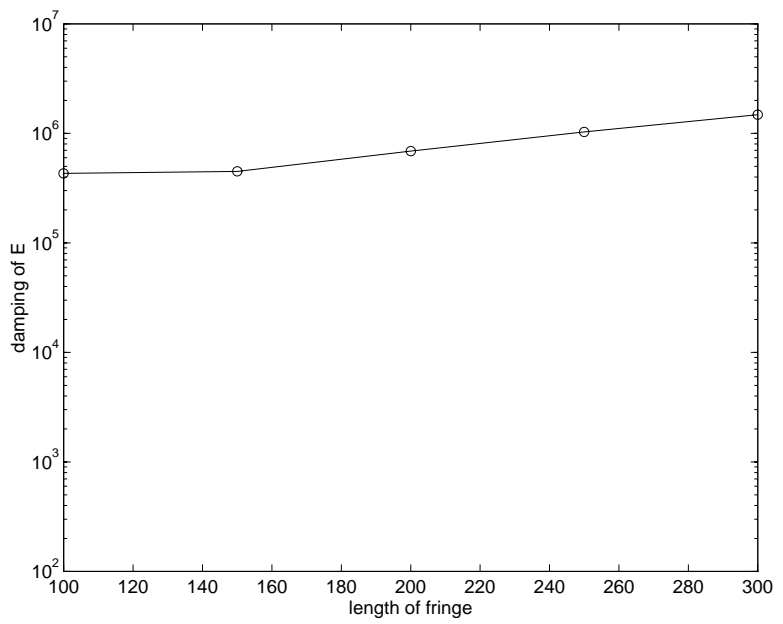

FIG. 6.3. Damping of the fringe region as a function of the length of the fringe region, simulations L through $P$.

with larger normal scales is expected. The results in Figure 6.1 and 6.2 are consistent with this theoretically predicted behavior: first a fast decay rate which increases with $\bar{\lambda}$ and then a substantially slower one. Figure 6.2 shows the total damping (the decrease of $E$ from the maximum value at $x \approx 350$ to the value of $E$ at $x=600$ ) in the fringe region as a function of $\bar{\lambda}$. The damping initially increases exponentially with $\bar{\lambda}$. This is also in agreement with the theoretical predictions above.

For the largest $\bar{\lambda}$ 's the total decay of the error decreases; see Figures 6.1 and 6.2. This phenomenon may be due to the fact that we are forcing toward a solution which is not an exact solution to the Navier-Stokes equations. Recall that the forcing function in the inflow problem is $F=-\bar{\lambda} \mathbf{w}^{1}+\bar{\lambda}\left(\mathbf{u}^{1}-\mathbf{u}^{1}\right)$. In the analysis above, 


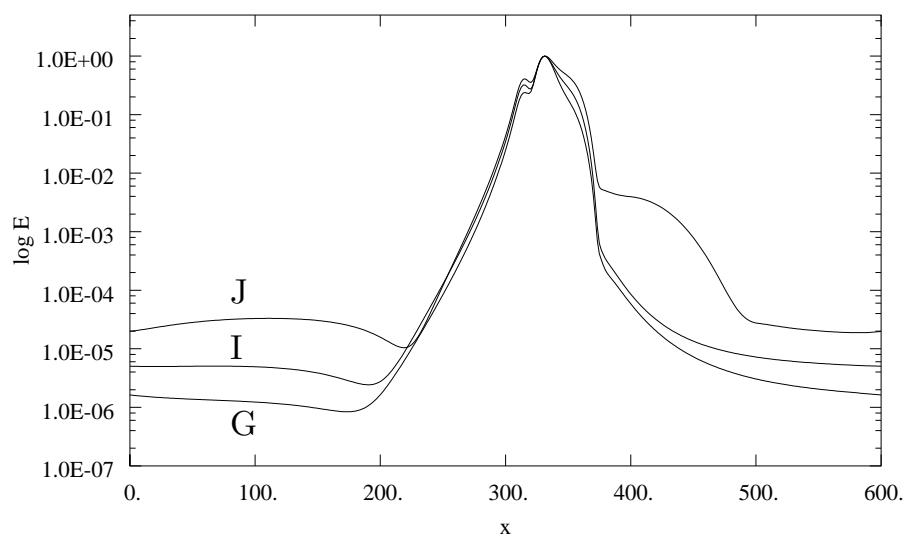

FIG. 6.4. Energy of the error vs. the streamwise distance. Variation with Reynolds number. Simulations $G, I$, and $J$. The maxima of all curves have been normalized to 1 .

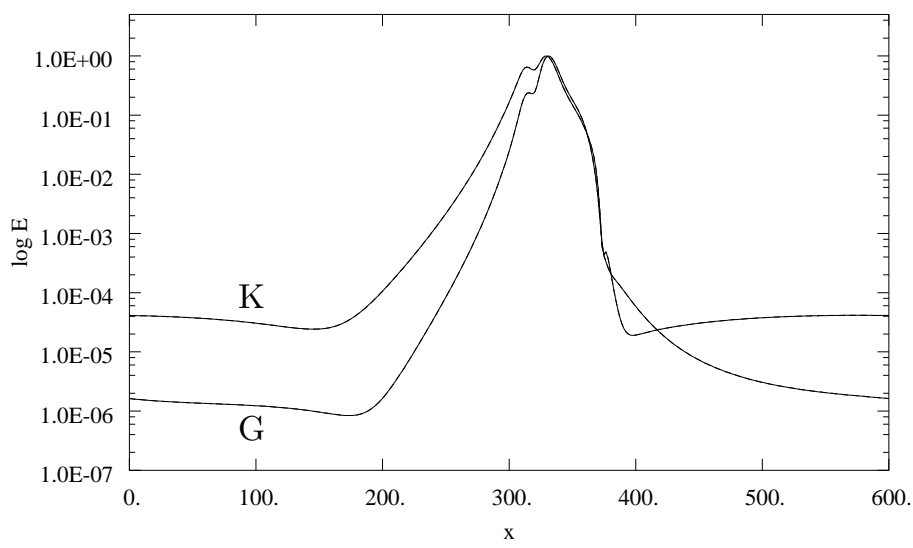

FIG. 6.5. Energy of the error vs. the streamwise distance. Variation with box height. Simulations $G$ and $K$. The maxima of all curves have been normalized to 1 .

$\dot{\mathbf{u}}^{1}=\mathbf{u}^{1}$ was assumed, which yields $F=-\bar{\lambda} \mathbf{w}^{1}$. If $\dot{\mathbf{u}}^{1}=\mathbf{u}_{B L}^{1} \neq \mathbf{u}^{1}$, an additional forcing term proportional to $\bar{\lambda}$ will remain in (4.3). That additional forcing term grows with $\bar{\lambda}$ and will lead to increasing errors in $|\mathbf{w}|$.

In practice one is interested in minimizing the length of the fringe region. Figure 6.3 shows that the damping decreases only slightly when the length of the fringe region is shortened to $1 / 3$ of the size used in the first set of numerical examples.

6.3. Theoretical and computational results: The outflow problem. The upstream influence of the fringe region is studied by considering the behavior of the error $E$ close to the outflow boundary of the physical domain, i.e., in the region $200<x<300$. Figure 6.4 shows the error for three different Reynolds numbers. The fact that the Blasius solution approximates the Navier-Stokes solution better for higher Reynolds numbers explains the decrease of the error in the physical domain with increasing Reynolds numbers. Figure 6.4 also shows that the upstream influence in relative terms is independent of the Reynolds number. Since the streamwise distance in each calculation is scaled with $\delta_{*}$ at the inflow boundary, this means that 
TABLE 6.3

Theoretically estimated upstream influence compared with the upstream influence in the numerical experiments shown in Figure 6.5.

\begin{tabular}{|c|c|c|c|}
\hline$y_{\max }$ & Decay rate $\left(\omega_{1}\right)$ & Decay rate $\left(\omega_{2}\right)$ & Estimated slope \\
\hline 10 & 0.1571 & 0.0772 & 0.054 \\
20 & 0.0785 & 0.0508 & 0.038 \\
\hline
\end{tabular}

the upstream influence in absolute terms increases proportionally to the Reynolds number. For the same reason, the height of the computational box and the fringe region parameters in (6.1) and (6.2) also increase in absolute terms with the Reynolds number.

The estimates in Theorem 5.2 and (5.32)-(5.34) show that the error with the largest normal scale has the slowest upstream decay. The velocity disturbances are proportional to $|\omega| \exp (|\omega|(x+\alpha))$, while the pressure disturbance is proportional to $\exp (|\omega|(x+\alpha))$. In fact, as the normal scale approaches infinity $(\omega \rightarrow 0)$ the upstream influence increases without bound (although the amplitude of the velocity disturbances goes to zero). In the simulations, the largest normal scale is proportional to the height of the computational box $y_{\max }$. Thus it is reasonable to assume that the increase of $y_{\max }$ in absolute terms with the Reynolds number is responsible for most of the increased upstream influence in Figure 6.4. Indeed, Figure 6.5 shows that this is the case. When $y_{\max }$ is doubled, the upstream influence substantially increases.

The theoretical analysis above does not account for the influence of the wall nor the finite height of the computational box. To quantitatively predict the upstream decay of the error, an estimate of $\omega_{\text {min }}$ is required. The slowest varying Fourier component that is zero at the wall and has a zero derivative at $y_{\max }$ has a wavelength of $4 y_{\max }$. Hence, a reasonable estimate of $\omega_{\min }$ would be $\pi /\left(2 y_{\max }\right)$. Another prediction of $\omega_{\min }$ can be obtained by solving the Orr-Sommerfeld equation for a steady mean flow given by the Blasius profile. For zero frequency disturbances (we consider a steady mean flow), the boundary conditions in the stability calculation correspond to the free-stream boundary conditions used in the simulation code. For a discussion of hydrodynamic instability and the Orr-Sommerfeld equation, see Drazin and Reid [20].

Table 6.3 shows a comparison between the upstream decay estimated using $\omega_{\min }=$ $\omega_{1}=\pi /\left(2 y_{\max }\right), \omega_{\min }=\omega_{2}$ corresponding to the least damped, zero frequency, upstream propagating mode from the Orr-Sommerfeld equation, and the decay obtained from Figure 6.5. The slope in Figure 6.5 has been estimated in the interval between the first peak in $\mathrm{E}$ and 100 units upstream of that peak. The decay rates have been computed using $y_{\max }=10$ and $y_{\max }=20$. The results using $\omega_{2}$ from the stability calculations and the numerical simulation correspond reasonably well whereas the estimate $\omega_{1}$ underpredicts the upstream influence.

Finally it should be mentioned that the height of the computational box is not the only factor that influences the upstream influence of the fringe region. As an example, Figure 6.1 shows that it is also influenced by the value of $\bar{\lambda}$. Traces of that influence can be seen in (5.24) and in the estimates (5.25)-(5.27), but in general there is not a complete explanation for that behavior in the simplified analysis presented above.

7. Summary and conclusions. An exact linear problem describing the deviation between an exact solution of the Navier-Stokes equations and the approximate solution computed using the fringe region technique was derived and energy estimates 
were obtained. However, the data could not be considered small; hence these estimates did not imply that the deviation was small. Moreover, only limited information about the spatial distribution of the deviation could be obtained from the linear problem. To facilitate a more detailed analysis, the linear problem was simplified and two constant coefficient half-plane problems describing the possibility to force the deviation toward zero inside the fringe region (the inflow problem) and the upstream influence of the fringe region (the outflow problem) were constructed. These problems were formally solved exactly and estimates of the deviation were obtained.

Theorem 5.1 indicates that it is possible to force the deviation toward zero in the fringe region. Large $|\omega|$ (corresponding to large $y$ derivatives) leads to a fast exponential decay of the deviation in the fringe region. For small $|\omega|$, the decay is small, but on the other hand, the deviation is of order $|\omega|$ in the velocities and pressure gradients while it is of order 1 in the pressure. It was also shown that the primary importance of the fringe function $\bar{\lambda}$ is to damp out the deviation associated with small $|\omega|$. Furthermore, it is important to know the exact solution in a region close to the inflow boundary. It is not sufficient to know $\mathbf{u}^{1}$ at one specific $x$ location.

Theorem 5.2 indicates that the upstream influence from the fringe region is small. Large $|\omega|$ leads to a fast exponential decay of the solution outside the fringe region. For small $|\omega|$, the decay is small, but on the other hand, the solution is of order $|\omega|$ in the velocities and pressure gradients while it is of order 1 in the pressure. The fringe function $\bar{\lambda}$ increases the upstream influence. Fortunately, $\bar{\lambda}$ is multiplied with $|\omega|$ in the estimates for the velocities and pressure gradients. This means that for $|\omega|$ small, the effect of $\bar{\lambda}$ is small. For large $|\omega|$, the value of $\bar{\lambda}$ is irrelevant due to the fast exponential decay of the solution.

Numerical computations using a Navier-Stokes code with the fringe region technique implemented were performed and comparisons with the theoretical results were done. In the inflow problem, the slow decay of the large normal scales for large $\bar{\lambda}$ predicted in Theorem 5.1 was verified. Furthermore, the exponential increase in damping of the fringe region due to the increase in $\bar{\lambda}$ was also verified. The importance of knowing an accurate solution in a region close to the inflow boundary was further stressed. The upstream influence predicted in Theorem 5.2 was also compared with computational results. By increasing the height of the computational box and thereby reducing the smallest normal wavenumber available, an increased upstream influence was obtained. The theoretical decay rates using estimates of $\omega_{\min }$ from Orr-Sommerfeld calculations and the decay rates from the Navier-Stokes calculations agreed reasonably well.

By combining the conclusions drawn from the analysis and numerical computations in this paper we arrive at the following. It is possible to force the periodic solution computed by the fringe region technique toward the exact solution. The lack of boundary conditions in the fringe region technique can be compensated by the knowledge of an exact solution to the incompressible Navier-Stokes equations in the fringe region of the computational domain. Moreover, the price one has to pay in the form of upstream influence from the fringe region is small. In short, the analysis and computations in this paper indicate that it is possible to use the fringe region technique in combination with the Fourier method to obtain accurate numerical solutions to the inflow-outflow problem associated with the incompressible Navier-Stokes equations.

Appendix A. The matrix exponential and coinciding eigenvalues. An explicit form of the solution given in (5.1) will be derived. For $\operatorname{Re}(s) \geq 0$ there is 
a double root at $\sigma_{2}=0$, i.e., $\lim \kappa_{4 \sigma_{2} \rightarrow 0}=-|\omega|=\kappa_{2}$. Let $\mathcal{M}_{s}=\mathbf{T D T}^{-1}$ and $\mathcal{M}_{d}=\mathbf{S K S}^{-1}$ be the matrix $\mathcal{M}$ for distinct and coinciding eigenvalues, respectively. The matrices $\mathbf{T}, \mathbf{D}, \mathbf{T}^{-\mathbf{1}}$ are

$$
\begin{gathered}
\mathbf{T}=\left(\begin{array}{cccc}
\mid & \mid & \mid & \mid \\
\psi_{1} & \psi_{3} & \psi_{2} & \psi_{4} \\
\mid & \mid & \mid & \mid
\end{array}\right), \quad \mathbf{D}=\left(\begin{array}{cccc}
|\omega| & 0 & 0 & 0 \\
0 & \kappa_{3} & 0 & 0 \\
0 & 0 & -|\omega| & 0 \\
0 & 0 & 0 & \kappa_{4}
\end{array}\right), \\
\mathbf{T}^{-1}=\left(\begin{array}{cccc}
\mid & \mid & \mid & \mid \\
\phi_{1} & \phi_{2} & \phi_{3} & \phi_{4} \\
\mid & \mid & \mid & \mid
\end{array}\right),
\end{gathered}
$$

$($ A.1 $) \psi_{1}=\left(\begin{array}{c}1 \\ i \frac{|\omega|}{\omega} \\ -\frac{\sigma_{1}}{\omega \mid} \\ i \omega\end{array}\right), \quad \psi_{3}=\left(\begin{array}{c}1 \\ i \frac{\kappa_{3}}{\omega} \\ 0 \\ i \frac{\kappa_{3}^{2}}{\omega}\end{array}\right), \quad \psi_{2}=\left(\begin{array}{c}1 \\ -i \frac{|\omega|}{\omega} \\ \frac{\sigma_{2}}{\omega \mid} \\ i \omega\end{array}\right), \quad \psi_{4}=\left(\begin{array}{c}1 \\ i \frac{\kappa_{4}}{\omega} \\ 0 \\ i \frac{\kappa_{4}^{2}}{\omega}\end{array}\right)$,

$(\mathrm{A} .2) \phi_{1}=\left(\begin{array}{c}-\frac{\epsilon \kappa_{3} \kappa_{4}}{2 \sigma_{1}} \\ \frac{\epsilon|\omega| \kappa_{4}\left(\kappa_{4}\left(\sigma_{1}-\sigma_{2}\right)+|\omega|\left(\sigma_{1}+\sigma_{2}\right)\right)}{2\left(\kappa_{3}-\kappa_{4}\right) \sigma_{1} \sigma_{2}} \\ -\frac{\epsilon \kappa_{3} \kappa_{4}}{2 \sigma_{2}} \\ \frac{-\epsilon|\omega| \kappa_{3}\left(\kappa_{3}\left(\sigma_{1}-\sigma_{2}\right)+|\omega|\left(\sigma_{1}+\sigma_{2}\right)\right)}{2\left(\kappa_{3}-\kappa_{4}\right) \sigma_{1} \sigma_{2}}\end{array}\right), \quad \phi_{2}=\left(\begin{array}{c}\frac{-i \epsilon \omega\left(\kappa_{3}+\kappa_{4}\right)}{2 \sigma_{1}} \\ \frac{-i \epsilon \omega\left(\sigma_{1}+\sigma_{2}\right)\left(\kappa_{4}^{2}-\omega^{2}\right)}{2\left(\kappa_{3}-\kappa_{4}\right) \sigma_{1} \sigma_{2}} \\ \frac{-i \epsilon \omega\left(\kappa_{3}+\kappa_{4}\right)}{2 \sigma_{2}} \\ \frac{i \epsilon \omega\left(\sigma_{1}+\sigma_{2}\right)\left(\kappa_{3}^{2}-\omega^{2}\right)}{2\left(\kappa_{3}-\kappa_{4}\right) \sigma_{1} \sigma_{2}}\end{array}\right)$,

$$
\phi_{3}=\left(\begin{array}{c}
-\frac{|\omega|}{2 \sigma_{1}} \\
\frac{\epsilon \omega^{2}\left(\kappa_{4}^{2}-\omega^{2}\right)}{\left(\kappa_{3}-\kappa_{4}\right) \sigma_{1} \sigma_{2}} \\
\frac{|\omega|}{2 \sigma_{2}} \\
-\frac{\epsilon \omega^{2}\left(\kappa_{3}^{2}-\omega^{2}\right)}{\left(\kappa_{3}-\kappa_{4}\right) \sigma_{1} \sigma_{2}}
\end{array}\right), \quad \phi_{4}=\left(\begin{array}{c}
\frac{i \epsilon \omega}{2 \sigma_{1}} \\
\frac{i \epsilon \omega\left(\kappa_{4}\left(\sigma_{1}+\sigma_{2}\right)+|\omega|\left(\sigma_{1}-\sigma_{2}\right)\right)}{2\left(\kappa_{3}-\kappa_{4}\right) \sigma_{1} \sigma_{2}} \\
\frac{i \epsilon \omega}{2 \sigma_{2}} \\
\frac{-i \epsilon \omega\left(\kappa_{3}\left(\sigma_{1}+\sigma_{2}\right)+|\omega|\left(\sigma_{1}-\sigma_{2}\right)\right)}{2\left(\kappa_{3}-\kappa_{4}\right) \sigma_{1} \sigma_{2}}
\end{array}\right) .
$$

The matrices $\mathbf{S}, \mathbf{K}, \mathbf{S}^{-1}$ are

$$
\begin{gathered}
\mathbf{S}=\left(\begin{array}{cccc}
\mid & \mid & \mid & \mid \\
\Psi_{1} & \Psi_{3} & \Psi_{2} & \Psi_{4} \\
\mid & \mid & \mid & \mid
\end{array}\right), \quad \mathbf{K}=\left(\begin{array}{cccc}
|\omega| & 0 & 0 & 0 \\
0 & \frac{\bar{u}+\epsilon|\omega|}{\epsilon} & 0 & 0 \\
0 & 0 & -|\omega| & 1 \\
0 & 0 & 0 & -|\omega|
\end{array}\right) \\
\mathbf{S}^{-1}=\left(\begin{array}{cccc}
\mid & \mid & \mid & \mid \\
\Phi_{1} & \Phi_{2} & \Phi_{3} & \Phi_{4} \\
\mid & \mid & \mid & \mid
\end{array}\right),
\end{gathered}
$$




$$
\begin{aligned}
& \Psi_{1}=\left(\begin{array}{c}
1 \\
i \frac{|\omega|}{\omega} \\
-2 \bar{u} \\
i \omega
\end{array}\right), \quad \Psi_{3}=\left(\begin{array}{c}
1 \\
i \frac{\bar{u}+\epsilon|\omega|}{\epsilon \omega} \\
0 \\
i \frac{(\bar{u}+\epsilon|\omega|)^{2}}{\epsilon^{2} \omega}
\end{array}\right) \\
& \Psi_{2}=\left(\begin{array}{c}
1 \\
-i \frac{|\omega|}{\omega} \\
0 \\
i \omega
\end{array}\right), \quad \Psi_{4}=\left(\begin{array}{c}
\frac{1}{|\omega|} \\
0 \\
\frac{\bar{u}+2 \epsilon|\omega|}{|\omega|} \\
-i \frac{\omega}{|\omega|}
\end{array}\right), \\
& \Phi_{1}=\left(\begin{array}{c}
\frac{\bar{u}+\epsilon|\omega|}{4 \bar{u}} \\
-\frac{\epsilon^{3}|\omega|^{3}}{\bar{u}(\bar{u}+2 \epsilon|\omega|)^{2}} \\
\frac{(\bar{u}+\epsilon|\omega|)(\bar{u}+4 \epsilon|\omega|)}{4(\bar{u}+2 \epsilon|\omega|)^{2}} \\
\frac{|\omega|(\bar{u}+\epsilon|\omega|)}{2(\bar{u}+2 \epsilon|\omega|)}
\end{array}\right), \quad \Phi_{2}=\left(\begin{array}{c}
\frac{-i \omega}{4|\omega|} \\
\frac{-i \epsilon|\omega| \omega}{(\bar{u}+2 \epsilon|\omega|)^{2}} \\
\frac{i \omega\left(3 \bar{u}^{2}+8 \bar{u} \epsilon|\omega|+8 \epsilon^{2} \omega^{2}\right)}{4|\omega|(\bar{u}+2 \epsilon|\omega|)^{2}} \\
\frac{-i \bar{u} \omega}{2(\bar{u}+2 \epsilon|\omega|)}
\end{array}\right), \\
& \Phi_{3}=\left(\begin{array}{c}
-\frac{1}{4 \bar{u}} \\
\frac{\epsilon^{2} \omega^{2}}{\bar{u}(\bar{u}+2 \epsilon|\omega|)^{2}} \\
-\frac{\bar{u}}{4(\bar{u}+2 \epsilon|\omega|)^{2}} \\
\frac{|\omega|}{2(\bar{u}+2 \epsilon|\omega|)}
\end{array}\right), \quad \Phi_{4}=\left(\begin{array}{c}
\frac{i \epsilon \omega}{4 \bar{u}|\omega|} \\
\frac{-i \epsilon^{2} \omega(\bar{u}+\epsilon|\omega|)}{\bar{u}(\bar{u}+2 \epsilon|\omega|)^{2}} \\
\frac{-i \epsilon|\omega|(3 \bar{u}+4 \epsilon|\omega|)}{4 \omega(\bar{u}+2 \epsilon|\omega|)^{2}} \\
\frac{i \epsilon \omega}{2(\bar{u}+2 \epsilon|\omega|)}
\end{array}\right) .
\end{aligned}
$$

A.1. Calculation of the matrix exponentials. Let $\mathbf{A}, \mathbf{B}, \mathbf{C}, \mathbf{D}, \mathbf{E}$ be square 4-by-4 matrices and $\mathbf{x}=(a, b, c, d)^{T}$ an arbitrary vector. Furthermore, let $\mathbf{A}=$ $\mathbf{B C B}^{-1}$ and $\mathbf{D E}=\mathbf{E D}$. The formulas

$$
e^{\mathbf{A}}=\sum_{i=0}^{\infty} \frac{\mathbf{A}^{i}}{i !}, \quad e^{\mathbf{A}}=\mathbf{B} e^{\mathbf{C}} \mathbf{B}^{-1}, \quad e^{\mathbf{D}+\mathbf{E}}=e^{\mathbf{D}} e^{\mathbf{E}},
$$

in the theory for matrix exponentials (see Golub and Van Loan [23]) lead to

$$
\begin{aligned}
e^{x \mathcal{M}_{s}} \mathbf{x} & =\mathbf{T} e^{x \mathbf{D}} \mathbf{T}^{-1} \mathbf{x}, \quad e^{x \mathcal{M}_{d}} \mathbf{x}=\mathbf{S} e^{x \mathbf{K}} \mathbf{S}^{-1} \mathbf{x}, \\
e^{x \mathbf{D}} & =\left(\begin{array}{cccc}
e^{|\omega| x} & 0 & 0 & 0 \\
0 & e^{\kappa_{3} x} & 0 & 0 \\
0 & 0 & e^{-|\omega| x} & 0 \\
0 & 0 & 0 & e^{\kappa_{4} x}
\end{array}\right) .
\end{aligned}
$$


Let $\mathbf{K}=\mathbf{K}_{1}+\mathbf{K}_{2}$, where

$$
\mathbf{K}_{1}=\left(\begin{array}{cccc}
|\omega| & 0 & 0 & 0 \\
0 & \frac{\bar{u}+\epsilon|\omega|}{\epsilon} & 0 & 0 \\
0 & 0 & -|\omega| & 0 \\
0 & 0 & 0 & -|\omega|
\end{array}\right), \quad \mathbf{K}_{2}=\left(\begin{array}{cccc}
0 & 0 & 0 & 0 \\
0 & 0 & 0 & 0 \\
0 & 0 & 0 & 1 \\
0 & 0 & 0 & 0
\end{array}\right) .
$$

It is easily verified that $\mathbf{K}_{1} \mathbf{K}_{2}=\mathbf{K}_{2} \mathbf{K}_{1}$. Equation (A.8) leads to $e^{x \mathbf{K}}=e^{x \mathbf{K}_{1}} e^{x \mathbf{K}_{2}}$, where $e^{x \mathbf{K}_{2}}=\mathbf{I}+x \mathbf{K}_{2}$ and

$$
\begin{gathered}
e^{x \mathbf{K}}=\left(\begin{array}{cccc}
e^{|\omega| x} & 0 & 0 & 0 \\
0 & e^{(\bar{u}+\epsilon|\omega|) x / \epsilon} & 0 & 0 \\
0 & 0 & e^{-|\omega| x} & x e^{-|\omega| x} \\
0 & 0 & 0 & e^{-|\omega| x}
\end{array}\right), \\
e^{x \mathbf{K}_{1}}=\left(\begin{array}{cccc}
e^{|\omega| x} & 0 & 0 & 0 \\
0 & e^{(\bar{u}+\epsilon|\omega|) x / \epsilon} & 0 & 0 \\
0 & 0 & e^{-|\omega| x} & 0 \\
0 & 0 & 0 & e^{-|\omega| x}
\end{array}\right), \quad e^{x \mathbf{K}_{2}}=\left(\begin{array}{cccc}
1 & 0 & 0 & 0 \\
0 & 1 & 0 & 0 \\
0 & 0 & 1 & x \\
0 & 0 & 0 & 1
\end{array}\right) .
\end{gathered}
$$

The final result in the single root case is

$$
e^{x \mathcal{M}_{s}} \mathbf{x}=\mathbf{T} e^{x \mathbf{D}} \mathbf{T}^{-1} \mathbf{x}=u_{1} \psi_{1} e^{|\omega| x}+u_{2} \psi_{3} e^{\kappa_{3} x}+u_{3} \psi_{2} e^{-|\omega| x}+u_{4} \psi_{4} e^{\kappa_{4} x},
$$

where

$$
\begin{gathered}
\mathbf{T} e^{x \mathbf{D}}=\left(\begin{array}{cccc}
\mid & \mid & \mid \\
\psi_{1} e^{|\omega| x} & \psi_{3} e^{\kappa_{3} x} & \psi_{2} e^{-|\omega| x} & \psi_{4} e^{\kappa_{4} x} \\
\mid & \mid & \mid & \mid
\end{array}\right), \\
\mathbf{T}^{-1} \mathbf{x}=a \phi_{1}+b \phi_{2}+c \phi_{3}+d \phi_{4}=\left(\begin{array}{l}
u_{1} \\
u_{2} \\
u_{3} \\
u_{4}
\end{array}\right) .
\end{gathered}
$$

The final result in the double root case is

$$
\begin{aligned}
e^{x \mathcal{M}_{d}} \mathbf{x} & =\mathbf{S} e^{x \mathbf{K}} \mathbf{S}^{-1} \mathbf{x} \\
& =v_{1} \Psi_{1} e^{|\omega| x}+v_{2} \Psi_{3} e^{(\bar{u}+\epsilon|\omega|) x / \epsilon}+\left(\left(v_{3} \Psi_{2}+v_{4} \Psi_{4}\right)+x v_{4} \Psi_{2}\right) e^{-|\omega| x},
\end{aligned}
$$

where

$$
\mathbf{S} e^{x \mathbf{K}}=\left(\begin{array}{cccc}
\mid & \mid & \mid & \mid \\
\Psi_{1} e^{|\omega| x} & \Psi_{3} e^{(\bar{u}+\epsilon|\omega|) x / \epsilon} & \Psi_{2} e^{-|\omega| x} & \left(\Psi_{4}+x \Psi_{2}\right) e^{-|\omega| x} \\
\mid & \mid & \mid & \mid
\end{array}\right)
$$

$$
\mathbf{S}^{-1} \mathbf{x}=a \Phi_{1}+b \Phi_{2}+c \Phi_{3}+d \Phi_{4}=\left(\begin{array}{c}
v_{1} \\
v_{2} \\
v_{3} \\
v_{4}
\end{array}\right)
$$


A.2. Convergence of the diagonal form to the Jordan form. It will be verified that

$$
e^{x \mathcal{M}_{s}} \mathbf{X} \stackrel{\sigma_{2} \rightarrow 0}{\rightarrow} e^{x \mathcal{M}_{d}} \mathbf{x}
$$

Consider (A.9) for small $\sigma_{2}$; a Taylor expansion yields

$$
e^{\kappa_{4} x}=\left(1-\frac{\sigma_{2}}{\bar{u}+2 \epsilon|\omega|} x\right) e^{-|\omega| x}+\mathcal{O}\left(\sigma_{2}^{2}\right)
$$

and consequently

$$
\begin{aligned}
\mathbf{T} e^{x \mathbf{D}} \mathbf{T}^{-1} \mathbf{x}= & u_{1} \psi_{1} e^{|\omega| x}+u_{2} \psi_{3} e^{\kappa_{3} x} \\
& +\left(\left(u_{3} \psi_{2}+u_{4} \psi_{4}\right)-x \frac{\sigma_{2}}{\bar{u}+2 \epsilon|\omega|} u_{4} \psi_{4}\right) e^{-|\omega| x} .
\end{aligned}
$$

Equations (A.17) and (A.12) imply that (A.15) holds if

$$
\begin{aligned}
u_{1} \psi_{1} \rightarrow v_{1} \Psi_{1}, \quad u_{2} \psi_{3} \rightarrow v_{2} \Psi_{3}, \quad u_{3} \psi_{2}+u_{4} \psi_{4} & \rightarrow v_{3} \Psi_{2}+v_{4} \Psi_{4}, \\
-\frac{\sigma_{2}}{\bar{u}+2 \epsilon|\omega|} u_{4} \psi_{4} & \rightarrow v_{4} \Psi_{2}
\end{aligned}
$$

as $\sigma_{2} \rightarrow 0$. Elementary but tedious algebra, the use of (A.1)-(A.3), (A.4)-(A.7), (A.10), (A.11), (A.14), and

$\kappa_{3}=\frac{\bar{u}+\epsilon|\omega|}{\epsilon}+\frac{\sigma_{2}}{\bar{u}+2 \epsilon|\omega|}+\mathcal{O}\left(\sigma_{2}^{2}\right), \quad \kappa_{4}=-|\omega|-\frac{\sigma_{2}}{\bar{u}+2 \epsilon|\omega|}+\mathcal{O}\left(\sigma_{2}^{2}\right), \quad \sigma_{1}=2 \bar{u}|\omega|+\sigma_{2}$

confirm that (A.18) and consequently also (A.15) hold.

A.3. The homogeneous and particular solution. The homogeneous part of the solution $\mathbf{W}_{h}$ in the single root case is given in (A.9) while its double root form is given in (A.12). It is possible to choose the coefficients $u_{i}$ in (A.9) as functions of the coefficients $v_{i}$ in (A.12) such that the single root form converges to the double root form. Let

$$
\left(\begin{array}{l}
u_{1} \\
u_{2} \\
u_{3} \\
u_{4}
\end{array}\right)=\left(\begin{array}{cccc}
1 & 0 & 0 & 0 \\
0 & 1 & 0 & 0 \\
0 & 0 & 1 & \frac{|\omega|(\bar{u}+2 \epsilon|\omega|)+\sigma_{2}}{|\omega| \sigma_{2}|\omega|} \\
0 & 0 & 0 & -\frac{u+2 \epsilon|\omega|}{\sigma_{2}}
\end{array}\right)\left(\begin{array}{c}
v_{1} \\
v_{2} \\
v_{3} \\
v_{4}
\end{array}\right) .
$$

Elementary algebra shows that (A.19) leads to (A.18); i.e., the single root solution converges to the double root solution as $\sigma_{2} \rightarrow 0$.

Equation (A.15) implies that the particular solution $\mathbf{W}_{p}$ in (5.1) can be determined by using $\mathbf{x}=\mathbf{F}(\bar{\lambda})$ in (A.9), (A.10), (A.11). The result is

$$
\begin{aligned}
\mathbf{W}_{p}(x)= & \int_{0}^{x} \bar{\lambda} a e^{-|\omega| \xi} d \xi e^{|\omega| x} \psi_{1}+\int_{0}^{x} \bar{\lambda} b e^{|\omega| \xi} d \xi e^{-|\omega| x} \psi_{2} \\
& +\int_{0}^{x} \bar{\lambda} c e^{-\kappa_{3} \xi} d \xi e^{\kappa_{3} x} \psi_{3}+\int_{0}^{x} \bar{\lambda} d e^{-\kappa_{4} \xi} d \xi e^{\kappa_{4} x} \psi_{4},
\end{aligned}
$$

where

$$
a=\frac{-|\omega| \widetilde{\widehat{\Delta u}}-i \omega \widetilde{\widehat{\Delta v}}}{2 \sigma_{1}}, \quad b=\frac{+|\omega| \widetilde{\widehat{\Delta u}}-i \omega \widetilde{\widehat{\Delta v}}}{2 \sigma_{2}},
$$




$$
\begin{aligned}
& c=+\frac{\epsilon \omega^{2}\left(\kappa_{4}^{2}-\omega^{2}\right)}{\left(\kappa_{3}-\kappa_{4}\right) \sigma_{1} \sigma_{2}} \widetilde{\widehat{\Delta u}}-i \omega \frac{\kappa_{4}\left(\sigma_{1}+\sigma_{2}\right)+2 \bar{u} \omega^{2}}{2\left(\kappa_{3}-\kappa_{4}\right) \sigma_{1} \sigma_{2}} \widetilde{\widehat{\Delta v}}, \\
& d=-\frac{\epsilon \omega^{2}\left(\kappa_{3}^{2}-\omega^{2}\right)}{\left(\kappa_{3}-\kappa_{4}\right) \sigma_{1} \sigma_{2}} \widetilde{\widehat{\Delta u}}+i \omega \frac{\kappa_{3}\left(\sigma_{1}+\sigma_{2}\right)+2 \bar{u} \omega^{2}}{2\left(\kappa_{3}-\kappa_{4}\right) \sigma_{1} \sigma_{2}} \widetilde{\widehat{\Delta v}} .
\end{aligned}
$$

Appendix B. Data considerations. Equation (4.5) leads to $\mathcal{L} \hat{g}_{t}=s \tilde{\hat{g}}(\omega, s)-$ $\hat{g}(\omega, 0)$. The first condition in (5.8) yields $\hat{g}(\omega, 0)=0$ and hence $\hat{g}_{t}=\mathcal{L}^{-1} s \tilde{\hat{g}}(\omega, s)$. The mean value theorem and (4.6) yield

$$
\begin{aligned}
\hat{u}_{0}(\omega, t) & =\hat{u}_{0}(0, t)+\frac{\partial \hat{u}_{0}(\theta \omega, t)}{\partial \omega} \omega, \\
\frac{\partial \hat{u}_{0}(\theta \omega, t)}{\partial \omega} & =\frac{1}{2 \pi} \int_{-\infty}^{\infty}(-i y) e^{-i \theta \omega y} u_{0}(y, t) d y, \quad 0<\theta<1 .
\end{aligned}
$$

The second condition in (5.8) leads to $\hat{u}_{0}(0, t)=0$. The third condition in (5.8) and (B.1) lead to

$$
\begin{aligned}
\left|\frac{\partial \hat{u}_{0}(\theta \omega, t)}{\partial \omega}\right| & \leq \frac{1}{2 \pi} \int_{-\delta}^{\delta}|y|\left|u_{0}(y, t)\right| d y \leq \frac{\delta^{2}}{\pi}\left|u_{0}(y, t)\right|_{m}, \\
\left|u_{0}(y, t)\right|_{m} & =\max _{-\delta \leq y \leq+\delta}\left|u_{0}(y, t)\right| .
\end{aligned}
$$

The second condition in (5.8) also yields $\int_{-\infty}^{+\infty}\left(u_{0}\right)_{t}(y, t) d y=0$; hence $\left(\hat{u}_{0}\right)_{t}$ can be estimated in a similar way. Let us summarize the results. Conditions (5.8) in physical space lead to

$$
\hat{g}_{t}(\omega, t)=\mathcal{L}^{-1} s \tilde{\hat{g}}(\omega, s), \quad\left|\frac{\partial^{j} \hat{u}_{0}(\omega, t)}{\partial t^{j}}\right| \leq \mathcal{C}_{0}\left|\frac{\partial^{j} u_{0}(y, t)}{\partial t^{j}}\right|_{m}|\omega|, \quad j=0,1,
$$

in transformed space. $\mathcal{C}_{0}$ is a constant.

The definition of the Fourier transform (4.6), (5.8), and (5.9) yield

$$
\begin{aligned}
\left|\omega^{r} \frac{\partial^{j} \hat{g}}{\partial t^{j}}\right| & \leq\left|\frac{1}{2 \pi} \int_{-\delta}^{\delta} e^{-i \omega y} \frac{\partial^{r+j} g}{\partial y^{r} \partial t^{j}} d y\right| \leq \mathcal{C}_{1}, \\
\left|\omega^{r} \widehat{\Delta \mathbf{u}}^{1}\right| & \leq\left|\frac{1}{2 \pi} \int_{-\delta}^{\delta} e^{-i \omega y} \frac{\partial^{r} \Delta \mathbf{u}^{1}}{\partial y^{r}} d y\right| \leq \mathcal{C}_{2}, \quad j=0,1,
\end{aligned}
$$

where $\mathcal{C}_{1}, \mathcal{C}_{2}$ are constants. The estimates in (B.2) with $r>0$ mean that $\hat{g}, \hat{g}_{t}$, and $\widehat{\Delta \mathbf{u}}^{1}$ decay fast as $|\omega| \rightarrow \infty$.

Appendix C. Proof of Theorem 3.1. Integration of $\left|\mathbf{w}^{1}\right|_{t}^{2}$ over the domain $\Omega_{+}=\left[x_{0}, x_{1}\right] \times\left[0, \infty\left[\right.\right.$ and frequent use of the continuity equations $\nabla \cdot \mathbf{u}^{1}=0, \nabla \cdot \mathbf{v}^{1}=$ $0, \nabla \cdot \mathbf{w}^{1}=0$ lead to

$$
\begin{aligned}
\left\|\mathbf{w}^{1}\right\|_{t}^{2} & +2 \epsilon\left(\left\|\mathbf{w}_{x}^{1}\right\|^{2}+\left\|\mathbf{w}_{y}^{1}\right\|^{2}\right) \\
= & -\underbrace{\int_{\Omega_{+}}\left(\mathbf{w}^{1}\right)^{T}\left(E+E^{T}\right) \mathbf{w}^{1}+2 \lambda\left(\left|\mathbf{w}^{1}\right|^{2}-\left(\mathbf{w}^{1}\right)^{T} \Delta \mathbf{u}^{1}\right) d x d y}_{I T}
\end{aligned}
$$




$$
\begin{gathered}
-\underbrace{2 \int_{0}^{\infty}\left[v_{1}\left(\frac{u^{2}+v^{2}}{2}\right)+u p-\epsilon\left(u u_{x}+v v_{x}\right)\right]_{x=x_{0}}^{x=x_{1}} d y}_{B T 1} \\
-\underbrace{2 \int_{x_{0}}^{x_{1}}\left[v_{2}\left(\frac{u^{2}+v^{2}}{2}\right)+v p-\epsilon\left(u u_{y}+v v_{y}\right)\right]_{y=0}^{y \rightarrow \infty} d x}_{B T 2} .
\end{gathered}
$$

$E^{T}+E$ is a real nonsingular symmetric matrix, which means that $I T$, the first term in (C.1), can be written

$$
\begin{aligned}
I T= & \int_{\Omega_{+}}\left[X^{T} \mathbf{w}^{1}\right]^{T}\left(\begin{array}{cc}
\lambda+\kappa_{1} & 0 \\
0 & \lambda+\kappa_{2}
\end{array}\right)\left[X^{T} \mathbf{w}^{1}\right] d x d y \\
& +\int_{\Omega_{+}} \lambda\left(\left|\mathbf{w}^{1}\right|^{2}-2\left(\mathbf{w}^{1}\right)^{T} \Delta \mathbf{u}^{1}\right) d x d y .
\end{aligned}
$$

The matrix $X$ has the eigenvectors of $E^{T}+E$ as columns and $\kappa_{1,2}= \pm \kappa$ where $\kappa$ is given in (3.2). With the exact solution $\mathbf{u}^{1}$ known in the left fringe region $\Omega_{1}$ (see Figures 2.1 and 2.3) we can put $\hat{\mathbf{u}}^{1}=\mathbf{u}^{1}$, which yields $\Delta \mathbf{u}^{1}=0$. In the right fringe region $\Omega_{3}, \Delta \mathbf{u}^{1} \neq 0$. This yields $-I T \leq-\tilde{\lambda}_{1}\left\|\mathbf{w}^{1}\right\|_{1}^{2}+\tilde{\lambda}_{2}\left\|\mathbf{w}^{1}\right\|_{2}^{2}+\tilde{\lambda}_{3}\left\|\Delta \mathbf{u}^{1}\right\|_{3}^{2}$.

$B T 2$ is identically zero since condition (2.2) leads to $\mathbf{w}^{1}=0$ at the wall $(y=0)$ and at infinity $(y \rightarrow \infty)$. The estimate $-B T 1 \leq\|g\|_{\Gamma}^{2}$ is obtained by using

$$
\begin{aligned}
& L_{0} \mathbf{w}=\left[\left(\begin{array}{ccc}
\left(v_{1}+\left|v_{1}\right|\right) / 2 & 0 & 1 \\
0 & \left(v_{1}+\left|v_{1}\right|\right) / 2 & 0
\end{array}\right)-\epsilon\left(\begin{array}{ccc}
1 & 0 & 0 \\
0 & 1 & 0
\end{array}\right) \frac{\partial}{\partial x}\right] \mathbf{w}=v_{1} g_{0}, \\
& L_{1} \mathbf{w}=\left[\left(\begin{array}{ccc}
\left(v_{1}-\left|v_{1}\right|\right) / 2 & 1 & 0 \\
0 & \left(v_{1}-\left|v_{1}\right|\right) / 2 & 0
\end{array}\right)-\epsilon\left(\begin{array}{lll}
1 & 0 & 0 \\
0 & 1 & 0
\end{array}\right) \frac{\partial}{\partial x}\right] \mathbf{w}=v_{1} g_{1}
\end{aligned}
$$

as boundary conditions. Nordström [21] and Hesthaven and Gottlieb [22] derived similar strongly well-posed boundary conditions for the compressible Navier-Stokes equations. The estimates of $I T, B T 1$, and $B T 2$ above and time integration of (C.1) conclude the proof of Theorem 3.1.

Appendix D. Proof of Theorem 5.1. Recall that $\mathcal{M}=\mathcal{M}(\bar{\lambda})$ and $\mathbf{F}=\mathbf{F}(0)=$ 0 . The solution is given by (5.6) with $a=b=c=d=0$. The boundary conditions in the inflow problem are

$$
\left(\begin{array}{c}
\tilde{\hat{u}} \\
\hat{\hat{v}}
\end{array}\right)(0)=\left(\begin{array}{c}
\tilde{\hat{u}}_{0} \\
\hat{\hat{v}}_{0}
\end{array}\right), \quad\left|\begin{array}{c}
\tilde{\hat{u}} \\
\hat{\hat{v}}
\end{array}\right|(x \rightarrow \infty)<\infty .
$$

The conditions at infinity yield $x_{1}=x_{3}=0$ while $x_{2}$ and $x_{4}$ are determined by

$$
\left(\begin{array}{c}
\tilde{\hat{u}}_{0} \\
\hat{\hat{v}}_{0}
\end{array}\right)=\left(\begin{array}{cc}
1 & 1 \\
-i|\omega| / \omega & i \kappa_{4} / \omega
\end{array}\right)\left(\begin{array}{c}
x_{2} \\
x_{4}
\end{array}\right)=\mathbf{E}_{s}\left(\begin{array}{c}
x_{2} \\
x_{4}
\end{array}\right) .
$$

Equation (D.1) determines $x_{2}, x_{4}$ uniquely if $\mathbf{E}_{s}$ is nonsingular. However,

$$
\lim _{\sigma_{2} \rightarrow 0} \operatorname{det}\left(\mathbf{E}_{s}\right)=\lim _{\sigma_{2} \rightarrow 0} \frac{i}{\omega}\left(\kappa_{4}+|\omega|\right)=0, \quad \sigma_{2}=s+\bar{\lambda}-\bar{u}|\omega|+i \omega \bar{v},
$$

which seems to imply that the inflow problem is not well-posed. 
At $\sigma_{2}=0$ there is a double root. The equation corresponding to (D.1) in the double root case is (see (A.19))

$$
\left(\begin{array}{c}
\tilde{\hat{u}}_{0} \\
\hat{\hat{v}}_{0}
\end{array}\right)=\left(\begin{array}{cc}
1 & 1 /|\omega| \\
-i|\omega| / \omega & 0
\end{array}\right)\left(\begin{array}{l}
y_{2} \\
y_{4}
\end{array}\right)=\mathbf{E}_{d}\left(\begin{array}{l}
y_{2} \\
y_{4}
\end{array}\right) .
$$

With a proper choice of constants $x_{2}, x_{4}$,

$$
\left(\begin{array}{l}
x_{2} \\
x_{4}
\end{array}\right)=\left(\begin{array}{cc}
1 & \left(|\omega|(\bar{u}+2 \epsilon|\omega|)+\sigma_{2}\right) /\left(|\omega| \sigma_{2}\right) \\
0 & -(\bar{u}+2 \epsilon|\omega|) / \sigma_{2}
\end{array}\right)\left(\begin{array}{l}
y_{2} \\
y_{4}
\end{array}\right)=\mathbf{A}\left(\begin{array}{l}
y_{2} \\
y_{4}
\end{array}\right),
$$

the single root solution converges to the double root solution as $\sigma_{2} \rightarrow 0$. With that choice, (D.1) transforms to

$$
\begin{aligned}
\left(\begin{array}{c}
\tilde{\hat{u}}_{0} \\
\hat{\hat{v}}_{0}
\end{array}\right) & =\mathbf{E}_{s} \mathbf{A}\left(\begin{array}{c}
y_{2} \\
y_{4}
\end{array}\right) \\
& =\left(\begin{array}{cc}
1 & 1 /|\omega| \\
-i|\omega| / \omega & -i\left\{(\bar{u}+2 \epsilon|\omega|)\left(\kappa_{4}+|\omega|\right)+\sigma_{2}\right\} /\left(\omega \sigma_{2}\right)
\end{array}\right)\left(\begin{array}{l}
y_{2} \\
y_{4}
\end{array}\right),
\end{aligned}
$$

where

$$
\lim _{\sigma_{2} \rightarrow 0} \mathbf{E}_{s} \mathbf{A}=\mathbf{E}_{d}, \quad \lim _{\sigma_{2} \rightarrow 0} \operatorname{det}\left(\mathbf{E}_{s} \mathbf{A}\right)=\operatorname{det}\left(\mathbf{E}_{d}\right)=\frac{i}{\omega} \neq 0 .
$$

By solving (D.3) for $y_{2}, y_{4}$ and transforming back to $x_{2}, x_{4}$ through the relation (D.2), we obtain

$$
\mathbf{W}(x)=\frac{\kappa_{4} \tilde{\hat{u}}_{0}+i \omega \tilde{\hat{v}}_{0}}{\kappa_{4}+|\omega|} \psi_{2} e^{-|\omega| x}+\frac{|\omega| \tilde{\hat{u}}_{0}-i \omega \tilde{\hat{v}}_{0}}{\kappa_{4}+|\omega|} \psi_{4} e^{\kappa_{4} x} .
$$

The solution (D.5) for each component $u, v, p$ can be written

$$
\begin{aligned}
\tilde{\hat{u}}= & e^{-|\omega| x}\left\{\left(1+\frac{|\omega|}{\Delta}\right) \tilde{\hat{u}}_{0}-i \frac{\omega}{\Delta} \tilde{\hat{v}}_{0}\right\}+e^{-(|\omega|+\Delta) x}\left\{-\frac{|\omega|}{\Delta} \tilde{\hat{u}}_{0}+i \frac{\omega}{\Delta} \tilde{\hat{v}}_{0}\right\}, \\
\tilde{\hat{v}}= & e^{-|\omega| x}\left\{-i \frac{|\omega|}{\omega}\left(1+\frac{|\omega|}{\Delta}\right) \tilde{\hat{u}}_{0}-\frac{|\omega|}{\Delta} \tilde{\hat{v}}_{0}\right\} \\
& +e^{-(|\omega|+\Delta) x}\left\{+i \frac{|\omega|}{\omega}\left(1+\frac{|\omega|}{\Delta}\right) \tilde{\hat{u}}_{0}+\left(1+\frac{|\omega|}{\Delta}\right) \tilde{\hat{v}}_{0}\right\}, \\
\tilde{\hat{p}}= & e^{-|\omega| x}\left\{\frac{\sigma}{|\omega|}\left(1+\frac{|\omega|}{\Delta}\right) \tilde{\hat{u}}_{0}-i \frac{\sigma}{\Delta} \frac{\omega}{|\omega|} \tilde{\hat{v}}_{0}\right\},
\end{aligned}
$$

where the notations $\Delta=\Delta_{4}, \sigma=\sigma_{2}, \kappa_{4}=-|\omega|-\Delta$ have been used. The definitions of $\kappa_{4}, \Delta_{4}$, and $\sigma_{2}$ are given by (5.2), (5.4), and (5.5) above.

Note that $\lim _{\sigma \rightarrow 0} \Delta=0$ indicates that the inflow solution might have a singularity at $\sigma=0$. However, a Taylor expansion yields

$$
\begin{aligned}
& \lim _{\sigma \rightarrow 0} \tilde{\hat{u}}=e^{-|\omega| x}\left((1+|\omega| x) \tilde{\hat{u}}_{0}-i \omega x \tilde{\hat{v}}_{0}\right), \\
& \lim _{\sigma \rightarrow 0} \tilde{\hat{v}}=e^{-|\omega| x}\left(-i \omega x \tilde{\hat{u}}_{0}+(1-|\omega| x) \tilde{\hat{v}}_{0}\right), \\
& \lim _{\sigma \rightarrow 0} \tilde{\hat{p}}=e^{-|\omega| x}(\bar{u}+2 \epsilon|\omega|)\left(\tilde{\hat{u}}_{0}-i \frac{\omega}{|\omega|} \tilde{\hat{v}}_{0}\right) .
\end{aligned}
$$


Note also that $\sigma(\omega=0)=s+\bar{\lambda} \neq 0$.

We need estimates of $\tilde{\hat{u}}, \tilde{\hat{v}}, \tilde{\hat{p}}$. Note that

$\operatorname{Re}(\sigma)>0 \Rightarrow \min _{\xi} \operatorname{Re}(\Delta)>0, \quad \operatorname{Re}(\sigma)<0 \Rightarrow \min _{\xi} \operatorname{Re}(\Delta)<0, \quad \operatorname{Re}(\sigma)=\eta+\bar{\lambda}-\bar{u}|\omega|$,

where $s=\eta+i \xi$. Let the function $\theta$ be defined by

$$
\theta=\min _{\xi}\left(|\omega|, \operatorname{Re}\left(\kappa_{4}\right)\right)=\left\{\begin{array}{ll}
|\omega|, & |\omega| \leq(\bar{\lambda}+\eta) / \bar{u} \\
|\omega|+\min _{\xi} \operatorname{Re}(\Delta), & |\omega|>(\bar{\lambda}+\eta) / \bar{u}
\end{array}\right\} .
$$

Equation (D.9) and estimates of (D.6)-(D.8) lead to

$$
\begin{aligned}
& |\tilde{\hat{u}}(x, \omega, s)| \leq \mathcal{K}_{1} e^{-\theta x}\left\{(1+|\omega|)\left|\tilde{\hat{u}}_{0}\right|+|\omega|\left|\tilde{\hat{v}}_{0}\right|\right\} \\
& |\tilde{\hat{v}}(x, \omega, s)| \leq \mathcal{K}_{2} e^{-\theta x}\left\{(1+|\omega|)\left|\tilde{\hat{u}}_{0}\right|+|\omega|\left|\tilde{\hat{v}}_{0}\right|\right\}
\end{aligned}
$$

$|\tilde{\hat{p}}(x, \omega, s)| \leq \mathcal{K}_{3} e^{-|\omega| x}$

$$
\times\left\{\left(\frac{1+|\omega|}{|\omega|}\right)\left(\left|s \tilde{\hat{u}}_{0}\right|+(1+|\omega|)\left|\tilde{\hat{u}}_{0}\right|\right)+\left|s \tilde{\hat{v}}_{0}\right|+(1+|\omega|)\left|\tilde{\hat{v}}_{0}\right|\right\},
$$

where the constants $\mathcal{K}_{1}, \mathcal{K}_{2}$, and $\mathcal{K}_{3}$ are of order 1 .

Parseval's relation and the estimates (D.10)-(D.12) lead to (5.13)-(5.15). To obtain the estimate (5.15), the first relation in (5.10) has been used. By the definition of the Fourier transform and the form of the solution in (D.8) we also get the estimate (5.16).

Appendix E. Proof of Theorem 5.2. Recall that $\mathcal{M}=\mathcal{M}(0)$ and $\mathbf{F}=\mathbf{F}(\bar{\lambda}) \neq$ 0 . The boundary conditions

$$
\left(\begin{array}{c}
\tilde{\hat{u}} \\
\hat{\hat{v}}
\end{array}\right)(0)=\left(\begin{array}{c}
\tilde{\hat{u}}_{0} \\
\tilde{\hat{v}}_{0}
\end{array}\right), \quad\left|\begin{array}{c}
\tilde{\hat{u}} \\
\tilde{\hat{v}}
\end{array}\right|(x \rightarrow-\infty)<\infty
$$

lead to

$$
\begin{aligned}
& x_{2}=-\int_{0}^{-\alpha} \bar{\lambda} b e^{|\omega| \xi} d \xi, \quad x_{4}=-\int_{0}^{-\alpha} \bar{\lambda} d e^{-\kappa_{4} \xi} d \xi, \\
& x_{1}=\frac{\left(\kappa_{3} \tilde{\hat{u}}_{0}+i \omega \tilde{\hat{v}}_{0}-\left(\kappa_{3}+|\omega|\right) x_{2}-\left(\kappa_{3}-\kappa_{4}\right) x_{4}\right)}{\kappa_{3}-|\omega|}, \\
& x_{3}=\frac{\left(-|\omega| \tilde{\hat{u}}_{0}-i \omega \tilde{\hat{v}}_{0}+2|\omega| x_{2}-\left(\kappa_{4}-|\omega|\right) x_{4}\right)}{\kappa_{3}-|\omega|},
\end{aligned}
$$

and the solution $\mathbf{W}(x)=\mathbf{W}_{h}(x)+\mathbf{W}_{p}(x) . \mathbf{W}_{h}(x)$ and $\mathbf{W}_{p}(x)$ are given in (5.23) and (5.24), respectively.

The solution outside the fringe region at $x=-\alpha-\beta, \beta>0$, can formally be written

$$
\mathbf{W}_{h}=A_{1} \psi_{1} e^{-|\omega|(\alpha+\beta)}+A_{3} \psi_{3} e^{-\kappa_{3}(\alpha+\beta)}, \quad \mathbf{W}_{p}=B_{1} \psi_{1} e^{-|\omega|(\alpha+\beta)}+B_{3} \psi_{3} e^{-\kappa_{3}(\alpha+\beta)},
$$


where

$$
\begin{aligned}
& A_{1}=+\frac{\kappa_{3} \tilde{\hat{u}}_{0}+i \omega \tilde{\hat{v}}_{0}}{\kappa_{3}-|\omega|}, \quad A_{3}=-\frac{|\omega| \tilde{\hat{u}}_{0}+i \omega \tilde{\hat{v}}_{0}}{\kappa_{3}-|\omega|}, \\
& B_{1}=\int_{0}^{-\alpha} \bar{\lambda} \frac{\left(\kappa_{3}+|\omega|\right) b e^{|\omega| \xi}+\left(\kappa_{3}-\kappa_{4}\right) d e^{-\kappa_{4} \xi}+\left(\kappa_{3}-|\omega|\right) a e^{-|\omega| \xi}}{\kappa_{3}-|\omega|} d \xi, \\
& B_{3}=\int_{0}^{-\alpha} \bar{\lambda} \frac{-2|\omega| b e^{|\omega| \xi}+\left(\kappa_{4}-|\omega|\right) d e^{-\kappa_{4} \xi}+\left(\kappa_{3}-|\omega|\right) c e^{-\kappa_{3} \xi}}{\kappa_{3}-|\omega|} d \xi .
\end{aligned}
$$

Note that the denominator $\kappa_{3}-|\omega|$ is nonzero for all $s, \omega$. It can be shown that $\left|A_{3} \psi_{3}\right|$ and $\left|B_{3} \psi_{3} e^{-\operatorname{Re}\left(\kappa_{3}\right) \alpha}\right|$ are bounded. Due to the fast exponential decay of $e^{-\operatorname{Re}\left(\kappa_{3}\right) \beta}$, $A_{3} \psi_{3} e^{-\kappa_{3}(\alpha+\beta)}$ can be neglected compared with $A_{1} \psi_{1} e^{-|\omega|(\alpha+\beta)}$ and $B_{3} \psi_{3} e^{-\kappa_{3}(\alpha+\beta)}$ can be neglected compared with $B_{1} \psi_{1} e^{-|\omega|(\alpha+\beta)}$.

Elementary but tedious algebra and the use of Parseval's relation lead to the estimates (5.25)-(5.30). To obtain the estimate (5.30), the first relation in (5.10) has been used. By the definition of the Fourier transform and the form of the solution in (5.24) we also get the estimate (5.31) of the pressure gradients.

Acknowledgment. We thank Anders Lundbladh for many fruitful discussions.

\section{REFERENCES}

[1] B. Gustafsson and H. O. Kreiss, Boundary conditions for time dependent problems with an artificial boundary, J. Comput. Phys., 30 (1979), pp. 333-351.

[2] D. Givoli, Non-reflecting boundary conditions, J. Comput. Phys., 94 (1991), pp. 1-29.

[3] H. O. Kreiss, Initial boundary value problems for hyperbolic systems, Comm. Pure Appl. Math., 23 (1970), pp. 277-298.

[4] B. Gustafsson, H. O. Kreiss, and A. Sundström, Stability theory of difference approximations for mixed initial boundary value problems II, Math. Comp., 26 (1972), pp. 649-686.

[5] P. R. Spalart, Direct numerical study of leading edge contamination, in Fluid Dynamics of Three-Dimensional Turbulent Shear Flows and Transition, AGARD-CP-438, 1988, pp. 5.1-5.13.

[6] F. P. Bertolotti, Th. Herbert, and P. R. Spalart, Linear and nonlinear stability of the Blasius boundary layer, J. Fluid Mech., 242 (1992), pp. 441-474.

[7] P. R. Spalart And J. H. Watmuff, Experimental and numerical study of a turbulent boundary layer with pressure gradients, J. Fluid Mech., 249 (1993), pp. 337-371.

[8] A. Lundbladh, P. J. Schmid, S. Berlin, and D. S. Henningson, Simulations of bypass transition for spatially evolving disturbances, in Proceedings of AGARD Symposium on Applications of Direct and Large-Eddy Simulation of Transition and Turbulence, AGARDCP-551, 1994, pp. 18.1-18.13.

[9] S. Berlin, A. Lundbladh, ANd D. S. Henningson, Spatial simulations of oblique transition in a boundary layer, Phys. Fluids, 6 (1994), pp. 1949-1951.

[10] M. HögBerg And D. S. Henningson, Secondary instability of cross-flow vortices in FalknerSkan-Cooke boundary layers, J. Fluid Mech., 368 (1998), pp. 339-357.

[11] M. Kloker, U. Konzelmann, and H. Fasel, Outflow boundary conditions for spatial NavierStokes simulations of transition boundary layers, AIAA J., 31 (1993), pp. 620-628.

[12] T. Colonious, S. K. Lele, And P. Moin, Boundary conditions for direct computations of aerodynamic sound generation, AIAA J., 31 (1993), pp. 1574-1582.

[13] S. KARNI, Accelerated convergence to steady state by gradual far-field damping, AIAA J., 30 (1992), pp. 1220-1228.

[14] J. Berenger, A perfectly matched layer for the absorption of electromagnetic waves, J. Comput. Phys., 114 (1994), pp. 185-200.

[15] H. C. Davies, Limitations of Some Common Lateral Boundary Schemes used in Regional NWP Models, Monthly Weather Review, 111 (1993), pp. 1002-1012.

[16] H. O. Kreiss and J. Lorenz, Initial-Boundary Value Problems and the Navier-Stokes Equations, Academic Press, New York, 1989.

[17] C. Johansson, Boundary conditions for open boundaries for the incompressible Navier-Stokes equation, J. Comput. Phys., 105 (1993), pp. 233-251. 
[18] J. Nordström, Accurate solutions of the Navier-Stokes equations despite unknown boundary data, J. Comput. Phys., 120 (1995), pp. 184-205.

[19] A. Lundbladh, D. S. Henningson, and A. V. Johansson, An Efficient Spectral Integration Method for the Solution of the Navier-Stokes Equations, FFA TN 1992-28, Bromma, Sweden, 1992 (unpublished).

[20] P. G. Drazin and W. H. Reid, Hydrodynamic Stability, Cambridge University Press, Cambridge, UK, 1981.

[21] J. NoRDSTRÖM, The use of characteristic boundary conditions for the Navier-Stokes equations, Comput. Fluids, 24 (1995), pp. 609-623.

[22] J. S. Hesthaven and D. GotTlieb, A stable penalty method for the compressible Navier-Stokes equations: I. Open boundary conditions, SIAM J. Sci. Comput., 17 (1996), pp. 579-612.

[23] G. H. Golub and C. F. Van LoAn, Matrix Computations, Johns Hopkins University Press, Baltimore, MD, 1983. 